\title{
Toric Initial Ideals of $\Delta$-Normal Configurations: Cohen-Macaulayness and Degree Bounds
}

\author{
EDWIN O'SHEA \\ oshea@math.washington.edu \\ REKHA R. THOMAS \\ thomas@math.washington.edu \\ Department of Mathematics, University of Washington, Seattle, WA 98195-4350
}

Received August 21, 2003; Revised June 9, 2004; Accepted June 28, 2004

\begin{abstract}
A normal (respectively, graded normal) vector configuration $\mathcal{A}$ defines the toric ideal $I_{\mathcal{A}}$ of a normal (respectively, projectively normal) toric variety. These ideals are Cohen-Macaulay, and when $\mathcal{A}$ is normal and graded, $I_{\mathcal{A}}$ is generated in degree at most the dimension of $I_{\mathcal{A}}$. Based on this, Sturmfels asked if these properties extend to initial ideals - when $\mathcal{A}$ is normal, is there an initial ideal of $I_{\mathcal{A}}$ that is Cohen-Macaulay, and when $\mathcal{A}$ is normal and graded, does $I_{\mathcal{A}}$ have a Gröbner basis generated in degree at most $\operatorname{dim}\left(I_{\mathcal{A}}\right)$ ? In this paper, we answer both questions positively for $\Delta$-normal configurations. These are normal configurations that admit a regular triangulation $\Delta$ with the property that the subconfiguration in each cell of the triangulation is again normal. Such configurations properly contain among them all vector configurations that admit a regular unimodular triangulation. We construct non-trivial families of both $\Delta$-normal and non- $\Delta$-normal configurations.
\end{abstract}

Keywords: toric ideals, triangulations, Hilbert bases, Gröbner bases

\section{Introduction}

A finite vector configuration $\mathcal{A}=\left\{\mathbf{a}_{i}: i=1, \ldots, n\right\} \subset \mathbb{Z}^{d}$ defines the toric ideal $I_{\mathcal{A}}:=$ $\left\langle\mathbf{x}^{\mathbf{u}}-\mathbf{x}^{\mathbf{v}}: \mathbf{u}, \mathbf{v} \in \mathbb{N}^{n}, \sum_{i=1}^{n} \mathbf{a}_{i} u_{i}=\sum_{i=1}^{n} \mathbf{a}_{i} v_{i}\right\rangle$ in the polynomial ring $R:=\mathbb{K}\left[x_{1}, \ldots\right.$, $\left.x_{n}\right]=\mathbb{K}[\mathbf{x}]$ where $\mathbb{K}$ is a field. Let cone $(\mathcal{A}), \mathbb{Z} \mathcal{A}$ and $\mathbb{N} \mathcal{A}$ denote the cone, lattice and semigroup spanned by the $\mathbb{R}_{\geq 0}, \mathbb{Z}$ and $\mathbb{N}$-linear combinations of $\mathcal{A}$ where $\mathbb{N}$ is the set of nonnegative integers. Let $\operatorname{dim}(\mathcal{A})$ be the Krull dimension of $R / I_{\mathcal{A}}$ which equals the rank of $\mathbb{Z} \mathcal{A}$. Assume $\operatorname{dim}(\mathcal{A})=d$. The configuration $\mathcal{A}$ is normal if $\mathbb{N} \mathcal{A}=\operatorname{cone}(\mathcal{A}) \cap \mathbb{Z} \mathcal{A}$ and graded if $\mathcal{A}$ spans an affine hyperplane in $\mathbb{R}^{d}$. A finite set $\mathcal{B} \subset \mathbb{Z}^{d}$ such that $\mathbb{N} \mathcal{B}=\operatorname{cone}(\mathcal{A}) \cap \mathbb{Z} \mathcal{A}$ is called a Hilbert basis of the semigroup cone $(\mathcal{A}) \cap \mathbb{Z} \mathcal{A}$. If $\mathcal{A}$ is normal, the zero set of $I_{\mathcal{A}}$ is a normal toric variety in $\mathbb{A}_{\mathbb{K}}^{n}$ of dimension $d$, and when $\mathcal{A}$ is also graded, it is a projectively normal toric variety in $\mathbb{P}_{\mathbb{K}}^{n-1}$ of dimension $d-1$. See [17] for details on toric ideals. A survey of recent results and open questions on normal configurations can be found in [2].

It is well known that initial ideals of a polynomial ideal inherit important invariants of the ideal such as dimension, degree and Hilbert function. Thus it is natural to ask if certain initial ideals inherit further properties of the ideal such as Cohen-Macaulayness, Betti numbers or reducedness (of the corresponding scheme). A result of Hochster [9] shows that when $\mathcal{A}$ is normal, $I_{\mathcal{A}}$ is Cohen-Macaulay. If $\mathcal{A}$ is also graded, then $I_{\mathcal{A}}$ is generated by homogeneous binomials of degree at most $d$ [17, Theorem 13.14]. Motivated by this, Sturmfels asked and conjectured the following. 
Question 1.1 If $\mathcal{A}$ is normal (more generally, if $I_{\mathcal{A}}$ is Cohen-Macaulay), is there a monomial initial ideal $\operatorname{in}_{\omega}\left(I_{\mathcal{A}}\right)$ of the toric ideal $I_{\mathcal{A}}$ that is Cohen-Macaulay?

Conjecture 1.2 ( [18, Conjecture 2.8]) If $\mathcal{A}$ is a graded, normal configuration then $I_{\mathcal{A}}$ has a Gröbner basis whose elements have degree at most $d=\operatorname{dim}(\mathcal{A})$.

In this paper, we show that Question 1.1 has a positive answer and Conjecture 1.2 is true when $\mathcal{A}$ is $\Delta$-normal. These configurations were defined by Hoşten and Thomas in [11]. We recall the definition. Let $\Delta$ be a pure $(d-1)$-dimensional simplicial complex with vertex set $[n]:=\{1, \ldots, n\}$. We denote the set of facets ( $d$-element faces) of $\Delta$ by max $\Delta$. For a set $\tau \subseteq[n]$, let $\mathcal{A}_{\tau}:=\left\{\mathbf{a}_{i} \in \mathcal{A}: i \in \tau\right\}$. We say that $\Delta$ is a triangulation of $\mathcal{A}$ if cone $(\mathcal{A})=\bigcup_{\sigma \in \max \Delta} \operatorname{cone}\left(\mathcal{A}_{\sigma}\right)$ and $\operatorname{cone}\left(\mathcal{A}_{\sigma_{i}}\right) \cap \operatorname{cone}\left(\mathcal{A}_{\sigma_{j}}\right)=\operatorname{cone}\left(\mathcal{A}_{\sigma_{i} \cap \sigma_{j}}\right)$ for all $\sigma_{i}, \sigma_{j} \in \max \Delta$. The Stanley-Reisner ideal of $\Delta$ is the squarefree monomial ideal $\left\langle\prod_{i \in \tau} x_{i}: \tau \notin \Delta\right\rangle \subseteq R$. A cornerstone in the combinatorics of toric initial ideals is the result by Sturmfels that the radical of a monomial initial ideal $\operatorname{in}_{\omega}\left(I_{\mathcal{A}}\right)$ of the toric ideal $I_{\mathcal{A}}$ is the Stanley-Reisner ideal of a triangulation $\Delta_{\omega}$ of $\mathcal{A}$ [17, Theorem. 8.3]. The ideal $\operatorname{in}_{\omega}\left(I_{\mathcal{A}}\right)$ is said to be supported on $\Delta_{\omega}$. Triangulations supporting initial ideals of $I_{\mathcal{A}}$ are the regular triangulations of $\mathcal{A}$. A triangulation $\Delta$ of $\mathcal{A}$ is unimodular if for each $\sigma \in \max \Delta$, $\mathbb{Z} \mathcal{A}_{\sigma}=\mathbb{Z} \mathcal{A}$.

Definition 1.3 If there exists a regular triangulation $\Delta$ of a configuration $\mathcal{A}$ such that for each $\sigma \in \max \Delta, \mathcal{A} \cap \operatorname{cone}\left(\mathcal{A}_{\sigma}\right)$ is a Hilbert basis of $\operatorname{cone}\left(\mathcal{A}_{\sigma}\right) \cap \mathbb{Z} \mathcal{A}$, we call $\mathcal{A}$ a $\Delta$-normal configuration.

Note that $\mathbb{Z} \mathcal{A}$ is used in the semigroups of Definition 1.3. All $\Delta$-normal configurations are normal. In Sections 3 and 4, we prove our main results.

Theorem 3.1 Let $\mathcal{A}$ be a $\Delta$-normal configuration. Then there exists a term order $\succ$ such that $\Delta=\Delta_{\succ}$ and $\operatorname{in}_{\succ}\left(I_{\mathcal{A}}\right)$ is Cohen-Macaulay.

Theorem 4.1 Let $\mathcal{A}$ be a graded $\Delta$-normal configuration. Then there exists a term order $\succ$ such that $\Delta=\Delta_{\succ}$ and the Gröbner basis of $I_{\mathcal{A}}$ with respect to $\succ$ consists of binomials of degree at most $d=\operatorname{dim}(\mathcal{A})$.

It was shown in [11] that if $\mathcal{A}$ is $\Delta$-normal then $I_{\mathcal{A}}$ has a monomial initial ideal that is free of embedded primes. In Section 2 we recall the main features of this initial ideal. Theorems 3.1 and 4.1 are proved by showing that this same initial ideal is Cohen-Macaulay and, when $\mathcal{A}$ is graded, generated in degree at most $d$. Our proofs are combinatorial and rely heavily on the structure of this initial ideal.

The set of $\Delta$-normal configurations is a proper subset of the set of normal configurations. They occur naturally in two ways. If $\mathcal{A}$ has a regular unimodular triangulation $\Delta$, then $\mathcal{A}$ is $\Delta$-normal. If cone $(\mathcal{A})$ is simplicial and we assume that its extreme rays are generated by $\mathbf{a}_{1}, \ldots, \mathbf{a}_{d}$, then $\mathcal{A}$ is $\Delta$-normal with respect to the coarsest regular triangulation $\Delta$ consisting of the unique facet $\sigma=\{1, \ldots, d\}$. These were the only examples known so far. Specific instances of normal configurations that are not $\Delta$-normal for any $\Delta$ are also 
known [11]. In Section 5 we construct non-trivial families of both $\Delta$-normal and non- $\Delta$ normal configurations.

Theorem 5.4 There are families of $\Delta$-normal configurations $\left\{\mathcal{A}^{d} \subset \mathbb{Z}^{d}, d \geq 5\right\}$ where cone $\left(\mathcal{A}^{d}\right)$ is non-simplicial and $\mathcal{A}^{d}$ has no regular unimodular triangulations.

Theorem 5.7 There is a family of normal, graded configurations $\left\{\mathcal{A}^{d} \subset \mathbb{Z}^{d}, d \geq 10\right\}$, that are not $\Delta$-normal for any regular triangulation $\Delta$.

\section{Background: An initial ideal without embedded primes}

We now recall from [11] the initial ideal of $I_{\mathcal{A}}$ without embedded primes when $\mathcal{A}$ is $\Delta$ normal. The construction uses the standard pair decomposition of a monomial ideal $M$ [19] which carries detailed information about $\operatorname{Ass}(M)$, the set of associated primes of $M$. Recall that every monomial prime ideal of $R$ is of the form $P_{\tau}:=\left\langle x_{j}: j \notin \tau\right\rangle$ for some $\tau \subseteq[n]$. The monomials of $R$ that do not lie in $M$ are the standard monomials of $M$. The support of a monomial $\mathbf{x}^{\mathbf{v}}$ is defined to be the support of its exponent vector $\mathbf{v}$ - i.e., $\operatorname{supp}\left(\mathbf{x}^{\mathbf{v}}\right)=\operatorname{supp}(\mathbf{v}):=\left\{i \in[n]: v_{i} \neq 0\right\}$.

Definition 2.1 ([19]) Let $M \subseteq R$ be a monomial ideal. For a standard monomial $\mathbf{x}^{\mathbf{u}}$ of $M$ and a set $\tau \subseteq[n],\left(\mathbf{x}^{\mathbf{u}}, \tau\right)$ is a pair of $M$ if all monomials in $\mathbf{x}^{\mathbf{u}} \cdot \mathbb{K}\left[x_{j}: j \in \tau\right]$ are standard monomials of $M$. We call $\left(\mathbf{x}^{\mathbf{u}}, \tau\right)$ a standard pair of $M$ if:

1. $\left(\mathbf{x}^{\mathbf{u}}, \tau\right)$ is a pair of $M$,

2. $\tau \cap \operatorname{supp}\left(\mathbf{x}^{\mathbf{u}}\right)=\emptyset$, and

3. the set of monomials in $\mathbf{x}^{\mathbf{u}} \cdot \mathbb{K}\left[x_{j}: j \in \tau\right]$ is not properly contained in the set of monomials in $\mathbf{x}^{\mathbf{v}} \cdot \mathbb{K}\left[x_{j}: j \in \tau^{\prime}\right]$ for any $\left(\mathbf{x}^{\mathbf{v}}, \tau^{\prime}\right)$ satisfying (1) and (2).

The set of standard pairs of $M$ is unique and is called the standard pair decomposition of $M$ since this set provides a decomposition of the standard monomials of $M$. The standard pair decomposition of $M$ is usually not a partition of the standard monomials of $M$. If $\mathbf{x}^{\mathbf{v}}$ is a standard monomial of $M$ then there is a standard pair $\left(\mathbf{x}^{\mathbf{u}}, \tau\right)$ of $M$ such that $\mathbf{x}^{\mathbf{u}}$ divides $\mathbf{x}^{\mathbf{v}}$ and $\operatorname{supp}\left(\mathbf{x}^{\mathbf{v}-\mathbf{u}}\right) \subseteq \tau$. In this case we say that $\mathbf{x}^{\mathbf{v}}$ is covered by $\left(\mathbf{x}^{\mathbf{u}}, \tau\right)$. We also use $\left(\mathbf{x}^{\mathbf{u}}, \tau\right)$ to denote the set of all standard monomials covered by it.

Theorem 2.2 (A) [19] Let $M$ be a monomial ideal in R. Then,

(1) $P_{\tau} \in \operatorname{Ass}(M)$ if and only if $M$ has a standard pair of the form $(*, \tau)$.

(2) $P_{\tau}$ is a minimal prime of $M$ if and only if $(1, \tau)$ is a standard pair of $M$. 
(B) [17] Let $M=\operatorname{in}_{\omega}\left(I_{\mathcal{A}}\right)$ be a monomial initial ideal of the toric ideal $I_{\mathcal{A}}$. Then,

(1) if $P_{\tau} \in \operatorname{Ass}(M)$ then $\tau$ is a face of the regular triangulation $\Delta_{\omega}$ of $\mathcal{A}$,

(2) $P_{\sigma}$ is a minimal prime of $M$ if and only if $\sigma \in \max \Delta_{\omega}$, and

(3) for $\sigma \in \max \Delta_{\omega}$, the number of standard pairs of $M$ of the form $(*, \sigma)$ is $\operatorname{vol}(\sigma)$, the normalized volume of $\sigma$ in $\Delta_{\omega}$.

We call $\mathbf{x}^{\mathbf{u}}$ and $\tau$ the root and face of the standard pair $\left(\mathbf{x}^{\mathbf{u}}, \tau\right)$. Let $\mathbf{A}$ (respectively, $\mathbf{A}_{\sigma}$ ) be the matrix whose set of columns is $\mathcal{A}$ (respectively, $\mathcal{A}_{\sigma}$ ). The normalized volume of $\sigma \in \max \Delta_{w}$ is the absolute value of the determinant of $\mathbf{A}_{\sigma}$ divided by the g.c.d. of the non-zero maximal minors of $\mathbf{A}$.

Theorem 2.3 ( [11, Theorem. 5]) Let $\mathcal{A}$ be a $\Delta$-normal configuration. Then there exists a term order $\succ$ such that $\Delta=\Delta_{\succ}$ and $\operatorname{in}_{\succ}\left(I_{\mathcal{A}}\right)$ is free of embedded primes.

The term order $\succ$ needed in Theorem 2.3 is described in [11] and is not directly used in this paper. The ideal $\operatorname{in}_{\succ}\left(I_{\mathcal{A}}\right)$ is shown to be free of embedded primes via an explicit description of its standard pairs. This structure is crucial for this paper and hence we recall it now. Assume without loss of generality that $\mathbb{Z} \mathcal{A}=\mathbb{Z}^{d}$. For $\sigma \in \max \Delta_{\succ}$, let $\mathrm{FP}_{\sigma}:=\left\{\sum_{i \in \sigma} \lambda_{i} \mathbf{a}_{i}: 0 \leq \lambda_{i}<1\right\}$ be the half open fundamental parallelopiped of $\operatorname{cone}\left(\mathcal{A}_{\sigma}\right)$. Then $\mathrm{FP}_{\sigma} \cap \mathbb{Z}^{d}$ has $\operatorname{vol}(\sigma)$ elements including the origin.

- For $\gamma \in \mathrm{FP}_{\sigma} \cap \mathbb{Z}^{d}$, let $\mathbf{x}^{\mathbf{u}_{\gamma}}$ be the cheapest monomial with respect to $\succ$ among all $\mathbf{x}^{\mathbf{u}} \in R$ with $\mathbf{A u}=\gamma$. It was shown in [11] that $\operatorname{supp}\left(\mathbf{x}^{\mathbf{u}_{\gamma}}\right) \subseteq \sigma_{\text {in }}:=\left\{i: \mathbf{a}_{i} \in \operatorname{cone}\left(\mathcal{A}_{\sigma}\right), i \notin \sigma\right\}$.

- The standard pairs of the initial ideal $\operatorname{in}_{\succ}\left(I_{\mathcal{A}}\right)$ in Theorem 2.3 are precisely the pairs $\left(\mathbf{x}^{\mathbf{u}_{\gamma}}, \sigma\right)$ as $\gamma$ varies in $\mathrm{FP}_{\sigma} \cap \mathbb{Z}^{d}$ for each $\sigma$ in $\max \Delta_{\succ}$. By Theorem 2.2, in ${ }_{\succ}\left(I_{\mathcal{A}}\right)$ is thus free of embedded primes.

For the remainder of this paper we will denote the term order of Theorem 2.3 by $\succ$, the toric initial ideal $\operatorname{in}_{\succ}\left(I_{\mathcal{A}}\right)$ of Theorem 2.3 by $J$ and its set of standard pairs by $\mathcal{S}(J)$. Other term orders will be denoted by $\omega$.

Example 2.4 Let $\mathcal{A}$ be the vector configuration consisting of the 13 columns of

$$
\mathbf{A}=\left(\begin{array}{lllllllllllll}
1 & 1 & 1 & 1 & 1 & 1 & 1 & 1 & 1 & 1 & 1 & 1 & 1 \\
0 & 1 & 2 & 3 & 0 & 1 & 2 & 3 & 0 & 1 & 2 & 3 & 0 \\
0 & 0 & 0 & 0 & 1 & 1 & 1 & 1 & 2 & 2 & 2 & 2 & 3
\end{array}\right)
$$

Then $\mathcal{A}$ is a graded supernormal configuration [10] which means that it is $\Delta$-normal with respect to every regular triangulation. Consider the regular triangulation

$$
\Delta=\{\{1,4,13\},\{4,11,12\},\{4,11,13\},\{11,12,13\}\}
$$

The configuration $\mathcal{A}$ and its regular triangulation $\Delta$ are shown in figure 1 . The toric ideal $I_{\mathcal{A}}$ lives in $R=\mathbb{K}[a, \ldots, m]$. In figure 1 , we have labeled the points of $\mathcal{A}$ by the variables 


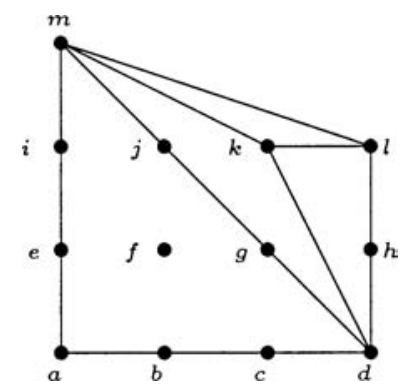

Figure 1. The graded supernormal configuration $\mathcal{A}$ of Example 2.4.

$a, \ldots, m$ corresponding to the columns of $\mathbf{A}$, instead of by $1, \ldots, 13$. The term order $\succ$ in Theorem 2.3 can be induced via the weight vector $(7,5,3,1,5,3,1,1,3,1,0,1,1)$ refined by the reverse lexicographic order with $b>e>c>f>i>g>j>$ $h>a>d>m>l>k$. The initial ideal (computed using Macaulay 2 [7]) is $J=$ $\left\langle j l, g l, h m, h^{2}, j^{2}, g j, i k, f k, i l, f l, j h, c l, g h, i h, c h, i j, f j, i g, e k, e l, b l, f h, g^{2}, c k\right.$, $b h, c g, e j, i^{2}, f i, c^{2}, a k, f^{2}, c i, e g, a l, e h, f g, c j, b k, h a, c f, b g, e i, b i, e f, b f, e c, b c$, $\left.e^{2}, b e, b^{2}, d m l\right\rangle \subset R$. Its standard pairs, grouped by the facets of $\Delta$ are:

\begin{tabular}{lc}
\hline faces & roots \\
\hline$\{1,4,13\}$ & $1, \mathrm{~b}, \mathrm{c}, \mathrm{e}, \mathrm{f}, \mathrm{g}, \mathrm{i}, \mathrm{j}, \mathrm{bj}$ \\
$\{4,11,13\}$ & $1, \mathrm{~g}, \mathrm{j}$ \\
$\{11,12,13\}$ & 1 \\
$\{4,11,12\}$ & $1, \mathrm{~h}$ \\
\hline
\end{tabular}

For $\sigma=\{1,4,13\}, \mathrm{FP}_{\sigma}$ consists of nine lattice points-Au for each exponent vector $\mathbf{u}$ of the roots $1, b, c, e, f, g, i, j, b j$. The last of these is $(2,2,2)^{t}$. The monomials $\mathbf{x}^{\mathbf{u}}$ of $R$ such that $\mathbf{A u}=(2,2,2)^{t}$ in increasing order with respect to $\succ$ are: $b j, e g, c i, f^{2}, a k$. Thus, $(b j,\{1,4,13\}) \in \mathcal{S}(J)$.

Remark 2.5 In general, the term order $\succ$ is constructed as in Example 2.4. When presented with a regular triangulation $\Delta$ for which $\mathcal{A}$ is $\Delta$-normal then choose a weight vector that induces the triangulation $\Delta$ such that no point is lifted higher than needs be to induce that triangulation. Namely, every lifted point is in a lower facet of the convex hull of the lifted configuration. Then break ties with any reverse lexicographic ordering with the vertices of $\Delta$ being cheaper than any non vertex of $\Delta$.

\section{Cohen-Macaulayness}

Theorem 3.1 Let $\mathcal{A}$ be a $\Delta$-normal configuration. Then there exists a term order $\succ$ such that $\Delta=\Delta_{\succ}$ and $\operatorname{in}_{\succ}\left(I_{\mathcal{A}}\right)$ is Cohen-Macaulay. 
This is done by showing that $J$ has a particular Stanley filtration [12] which implies Cohen-Macaulayness $[12,15]$. Stanley filtrations are special Stanley decompositions.

Definition 3.2 Let $M \subseteq R$ be a monomial ideal. A Stanley decomposition of $M$ is a set of pairs of $M,\left\{\left(\mathbf{x}^{\mathbf{u}}, \tau\right)\right\}$, that partition the standard monomials of $M$.

Remark 3.3 Every monomial ideal $M$ has the trivial Stanley decomposition $\left\{\left(\mathbf{x}^{\mathbf{u}}, \varnothing\right)\right.$ : $\left.\mathbf{x}^{\mathbf{u}} \notin M\right\}$. There can be many Stanley decompositions of a monomial ideal.

We will show that the standard pair decomposition $\mathcal{S}(J)$ of $J$ can be modified first to a Stanley decomposition and then to a Stanley filtration of the needed form. For $\tau \subseteq[n]$ let $\pi_{\tau}: R \rightarrow R_{\tau}:=\mathbb{K}\left[x_{i}: i \notin \tau\right]$ be the projection map where $\pi_{\tau}\left(x_{i}\right)=x_{i}$ if $i \notin \tau$ and $\pi_{\tau}\left(x_{i}\right)=1$ if $i \in \tau$.

Theorem 3.4 ([17, Section 12.D]) If $\sigma \in \max \Delta_{\omega}$ for a regular triangulation $\Delta_{\omega}$ of $\mathcal{A}$, then $\pi_{\sigma}\left(\operatorname{in}_{\omega}\left(I_{\mathcal{A}}\right)\right)$ is an artinian monomial ideal in $R_{\sigma}$ with $\operatorname{vol}(\sigma)$-many standard monomials which are precisely the roots of the standard pairs $(*, \sigma)$ of $\operatorname{in}_{\omega}\left(I_{\mathcal{A}}\right)$.

Corollary 3.5 If $\left(\mathbf{x}^{\mathbf{u}}, \sigma\right)$ is a standard pair in $\mathcal{S}(J)$, then every divisor of $\mathbf{x}^{\mathbf{u}}$ is also the root of a standard pair in $\mathcal{S}(J)$.

Lemma 3.6 Let $\left(\mathbf{x}^{\mathbf{u}}, \sigma\right)$ and $\left(\mathbf{x}^{\mathbf{v}}, \tau\right)$ be two standard pairs in $\mathcal{S}(J)$. If $\mathbf{x}^{\mathbf{u}} \neq \mathbf{x}^{\mathbf{v}}$ then $\left(\mathbf{x}^{\mathbf{u}}, \sigma\right) \cap\left(\mathbf{x}^{\mathbf{v}}, \tau\right)=\emptyset$.

Proof: $\quad$ Suppose $\mathbf{x}^{\mathbf{m}} \in\left(\mathbf{x}^{\mathbf{u}}, \sigma\right) \cap\left(\mathbf{x}^{\mathbf{v}}, \tau\right)$. Then $\mathbf{x}^{\mathbf{m}}=\mathbf{x}^{\mathbf{u}} \mathbf{x}^{\mathbf{m}_{\sigma}}=\mathbf{x}^{\mathbf{v}} \mathbf{x}^{\mathbf{m}_{\tau}}$ with $\operatorname{supp}\left(\mathbf{x}^{\mathbf{m}_{\sigma}}\right) \subseteq \sigma$, $\operatorname{supp}\left(\mathbf{x}^{\mathbf{m}_{\tau}}\right) \subseteq \tau$ and $\operatorname{supp}\left(\mathbf{x}^{\mathbf{u}}\right), \operatorname{supp}\left(\mathbf{x}^{\mathbf{v}}\right)$ outside the vertices of $\Delta_{\succ}$ and thus in particular, outside $\sigma \cup \tau$. Hence, $\mathbf{x}^{\mathbf{u}}=\mathbf{x}^{\mathbf{v}}$.

Corollary 3.7 If $\mathcal{A}$ is normal, cone $(\mathcal{A})$ is simplicial (generated without loss of generality by $\left.\mathbf{a}_{1}, \ldots, \mathbf{a}_{d}\right)$, and $J$ is the special initial ideal of Theorem 2.3 supported on $\Delta_{\succ}=$ $\{\{1, \ldots, d\}\}$, then $\mathcal{S}(J)$ is a Stanley decomposition.

Proof: Here $\mathcal{A}$ is $\Delta_{\succ}$-normal. All standard pairs in $\mathcal{S}(J)$ have face $[d]$ and roots the standard monomials of $\pi_{[d]}(J)$ and hence distinct and so, by Lemma 3.6, no two standard pairs intersect.

However, if $\left|\max \Delta_{\succ}\right|>1$, then it is precisely the standard pairs in $\mathcal{S}(J)$ with a common root that $\operatorname{stop} \mathcal{S}(J)$ from being a partition. Such pairs always exist when $\left|\max \Delta_{\succ}\right|>1$-for instance, $(1, \sigma)$ is in $\mathcal{S}(J)$ for all $\sigma \in \max \Delta_{\succ}$. We will use the combinatorial notion of shellings to create new pairs that partition the standard monomials covered by each set of standard pairs with a common root. In Section 2 we defined $\sigma_{\text {in }}:=\left\{i: \mathbf{a}_{i} \in \operatorname{cone}\left(\mathcal{A}_{\sigma}\right), i \notin\right.$ $\sigma\}$. The following lemma identifies the faces in all standard pairs that share a root.

Lemma 3.8 If $\mathbf{x}^{\mathbf{u}}$ is a root of a standard pair in $\mathcal{S}(J)$, then $\left\{\sigma \in \max \Delta_{\succ}:\left(\mathbf{x}^{\mathbf{u}}, \sigma\right) \in\right.$ $\mathcal{S}(J)\}=\left\{\sigma \in \max \Delta_{\succ}: \operatorname{supp}\left(\mathbf{x}^{\mathbf{u}}\right) \subseteq \sigma_{\text {in }}\right\}$. 
Proof: Recall from Section 2 that if $\left(\mathbf{x}^{\mathbf{u}}, \sigma\right) \in \mathcal{S}(J)$, then $\operatorname{supp}\left(\mathbf{x}^{\mathbf{u}}\right) \subseteq \sigma_{\text {in. }}$. Conversely, suppose $\left(\mathbf{x}^{\mathbf{u}}, \tau\right)$ is a standard pair in $\mathcal{S}(J)$ and $\operatorname{supp}\left(\mathbf{x}^{\mathbf{u}}\right) \subseteq \sigma_{\text {in }}$ for some $\sigma \neq \tau$ in $\max \Delta_{\succ}$. Then $\operatorname{supp}\left(\mathbf{x}^{\mathbf{u}}\right) \subseteq \tau_{\text {in }} \cap \sigma_{\text {in }}=(\tau \cap \sigma)_{\text {in }}$. Then Au $\in \operatorname{cone}\left(\mathcal{A}_{\sigma \cap \tau}\right)$. Since Au $\in \mathrm{FP}_{\tau} \cap \mathbb{Z}^{d} \cap$ $\operatorname{cone}\left(\mathcal{A}_{\sigma \cap \tau}\right)$, it is also in $\mathrm{FP}_{\sigma} \cap \mathbb{Z}^{d}$. Further, $\mathbf{x}^{\mathbf{u}}$ is the cheapest monomial with respect to $\succ$ among all monomials $\mathbf{x}^{\mathbf{v}}$ in $R$ with $\mathbf{A u}=\mathbf{A v}$. This implies that $\left(\mathbf{x}^{\mathbf{u}}, \sigma\right)$ is also a standard pair of $J$.

Definition 3.9 ( [16, Chapter 3, Definition 2.1]) Let $\mathcal{C}$ be a pure simplicial complex. A shelling of $\mathcal{C}$ is a linear ordering $F_{1}, \ldots, F_{s}$ of the facets of $\mathcal{C}$ such that for each $j, 1<j \leq s$, the collection of faces of $\mathcal{C}$ supported in $\left(F_{1} \cup \cdots \cup F_{j}\right) \backslash\left(F_{1} \cup \cdots \cup F_{j-1}\right)$ has a unique minimal face. A simplicial complex $\mathcal{C}$ is shellable if it has a shelling.

Let $F$ be a face of a simplicial complex $\mathcal{C}$. Then $\operatorname{star}(F, \mathcal{C}):=\{G \in \mathcal{C}: F \cup G \in \mathcal{C}\}$ is the simplicial complex generated by all $G \in \mathcal{C}$ containing $F$. We sometimes write $\operatorname{star}(F)$ for $\operatorname{star}(F, \mathcal{C})$ when $\mathcal{C}$ is obvious. The following is a mild generalization of Lemma 8.7 in [20].

Lemma 3.10 Let $\mathcal{C}$ be a pure shellable simplicial complex with shelling order $F_{1}, \ldots, F_{s}$. If $F$ is any face of $\mathcal{C}$ then the restriction of the global shelling order to $\operatorname{star}(F, \mathcal{C})$ yields a shelling of $\operatorname{star}(F, \mathcal{C})$.

Definition 3.11 For a $\operatorname{root} \mathbf{x}^{\mathbf{u}}$ in $\mathcal{S}(J)$, let $\delta\left(\mathbf{x}^{\mathbf{u}}\right):=\bigcap\left\{\sigma:\left(\mathbf{x}^{\mathbf{u}}, \sigma\right) \in \mathcal{S}(J)\right\}$.

In the following arguments we fix a root $\mathbf{x}^{\mathbf{u}}$ of a standard pair in $\mathcal{S}(J)$. By Lemma 3.8, $\delta\left(\mathbf{x}^{\mathbf{u}}\right)=\bigcap\left\{\sigma \in \max \Delta_{\succ}: \operatorname{supp}\left(\mathbf{x}^{\mathbf{u}}\right) \subseteq \sigma_{\text {in }}\right\}$ and

$$
\max \operatorname{star}\left(\delta\left(\mathbf{x}^{\mathbf{u}}\right), \Delta_{\succ}\right)=\left\{\sigma:\left(\mathbf{x}^{\mathbf{u}}, \sigma\right) \in \mathcal{S}(J)\right\}=\left\{\sigma \in \max \Delta_{\succ}: \operatorname{supp}\left(\mathbf{x}^{\mathbf{u}}\right) \subseteq \sigma_{\text {in }}\right\} .
$$

If $\mathbf{x}^{\mathbf{v}}$ divides $\mathbf{x}^{\mathbf{u}}$, then $\operatorname{star}\left(\delta\left(\mathbf{x}^{\mathbf{u}}\right)\right) \subseteq \operatorname{star}\left(\delta\left(\mathbf{x}^{\mathbf{v}}\right)\right)$. The regular triangulation $\Delta_{\succ}$ is shellable [20]. In the rest of this section, we fix a shelling of $\Delta_{\succ}$. By Lemma 3.10, this induces a shelling of $\operatorname{star}\left(\delta\left(\mathbf{x}^{\mathbf{u}}\right)\right)$. Let us assume without loss of generality that $\sigma_{1}, \sigma_{2}, \ldots, \sigma_{t}$ is the induced shelling order of the facets of $\max \operatorname{star}\left(\delta\left(\mathbf{x}^{\mathbf{u}}\right)\right)$. For $\sigma_{j} \in \max \operatorname{star}\left(\delta\left(\mathbf{x}^{\mathbf{u}}\right)\right)$, let $Q_{\mathbf{u}}^{\sigma_{j}}$ be the unique minimal face in $\operatorname{star}\left(\delta\left(\mathbf{x}^{\mathbf{u}}\right)\right)$ as described in Definition 3.9. It is known [20] that $Q_{\mathbf{u}}^{\sigma_{j}}=\left\{v \in \sigma_{j}: \sigma_{j} \backslash\{v\} \subseteq \sigma_{l}\right.$ for some $\left.l<j\right\}$. For each $Q_{\mathbf{u}}^{\sigma_{j}}$ define an interval $I_{\mathbf{u}}^{\sigma_{j}}:=$ $\left\{F: Q_{\mathbf{u}}^{\sigma_{j}} \subseteq F \subseteq \sigma_{j}\right\}$

Lemma 3.12 ([20, pp. 247]) The simplicial complex $\operatorname{star}\left(\delta\left(\mathbf{x}^{\mathbf{u}}\right)\right)$ is the disjoint union of the intervals $I_{\mathbf{u}}^{\sigma_{j}}, j=1, \ldots, t$.

Example 2.4 (continued) Consider the root $g$ of $\mathcal{S}(J)$ and the shelling order $\sigma_{1}=$ $\{4,11,12\}, \sigma_{2}=\{11,12,13\}, \sigma_{3}=\{4,11,13\}$ and $\sigma_{4}=\{1,4,13\}$ on $\Delta_{\succ}$. Then $\sigma_{3}, \sigma_{4}$ is the induced shelling order of $\operatorname{star}(\delta(g))$. From this we attain the sets $Q_{g}^{\sigma_{3}}=\emptyset$ and $Q_{g}^{\sigma_{4}}=\{1\}$ and the intervals $I_{g}^{\sigma_{3}}=\{F: F \subseteq\{4,11,13\}\}$ and $I_{g}^{\sigma_{4}}=\{F:\{1\} \subseteq F \subseteq\{1,4,13\}\}$. Clearly, $I_{g}^{\sigma_{3}} \cup I_{g}^{\sigma_{4}}$ partitions star $(\delta(g))$ which is the simplicial complex generated by all faces of $\{4,11,13\}$ and $\{1,4,13\}$. 
Remark 3.13 Note that by construction, the partial union $I_{\mathbf{u}}^{\sigma_{1}} \cup I_{\mathbf{u}}^{\sigma_{2}} \cup \cdots \cup I_{\mathbf{u}}^{\sigma_{l}}$ is a partition of the subcomplex with maximal faces $\sigma_{1}, \sigma_{2}, \ldots, \sigma_{l}$ and that $Q_{\mathbf{u}}^{\sigma_{j}}$ is not contained in this partial union for any $j>l$.

Definition 3.14 For a root $\mathbf{x}^{\mathbf{u}}$ in $\mathcal{S}(J)$ and a facet $\sigma \in \max \Delta_{\succ}$ define the monomial

$$
m_{\mathbf{u}}^{\sigma}:= \begin{cases}1 & \text { if } \sigma \in \operatorname{star}\left(\delta\left(\mathbf{x}^{\mathbf{u}}\right)\right), Q_{\mathbf{u}}^{\sigma}=\emptyset \\ \prod_{l \in Q_{\mathbf{u}}^{\sigma}} x_{l} & \text { if } \sigma \in \operatorname{star}\left(\delta\left(\mathbf{x}^{\mathbf{u}}\right)\right), Q_{\mathbf{u}}^{\sigma} \neq \emptyset \\ 1 & \text { otherwise. }\end{cases}
$$

Recall that we have fixed a shelling of $\Delta_{\succ}$ and thus, by Lemma 3.10, a shelling of $\operatorname{star}\left(\delta\left(\mathbf{x}^{\mathbf{u}}\right)\right)$ for each root $\mathbf{x}^{\mathbf{u}}$ in $\mathcal{S}(J)$. Therefore, if $\sigma \in \max \operatorname{star}\left(\delta\left(\mathbf{x}^{\mathbf{u}}\right)\right), Q_{\mathbf{u}}^{\sigma}$ is uniquely defined. We return to the fixed $\operatorname{root} \mathbf{x}^{\mathbf{u}}$ and the shelling $\sigma_{1}, \ldots, \sigma_{t}$ of $\operatorname{star}\left(\delta\left(\mathbf{x}^{\mathbf{u}}\right)\right)$.

Lemma 3.15 The standard monomials of $J$ in $\bigcup_{j=1}^{t}\left(\mathbf{x}^{\mathbf{u}}, \sigma_{j}\right)$ are partitioned by the pairs $\left(\mathbf{x}^{\mathbf{u}} \cdot m_{\mathbf{u}}^{\sigma_{j}}, \sigma_{j}\right), j=1, \ldots, t$.

Proof: By Lemma 3.12, $I_{\mathbf{u}}^{\sigma_{1}} \cup I_{\mathbf{u}}^{\sigma_{2}} \cup \cdots \cup I_{\mathbf{u}}^{\sigma_{t}}$ is a partition of $\operatorname{star}\left(\delta\left(\mathbf{x}^{\mathbf{u}}\right)\right)$. Hence, if $\mathbf{x}^{\mathbf{v}} \in \bigcup_{j=1}^{t}\left(\mathbf{x}^{\mathbf{u}}, \sigma_{j}\right)$ then $\operatorname{supp}\left(\mathbf{x}^{\mathbf{v}-\mathbf{u}}\right) \in I_{\mathbf{u}}^{\sigma_{j}}$ for a unique $I_{\mathbf{u}}^{\sigma_{j}}$. By construction, $I_{\mathbf{u}}^{\sigma_{j}}=$ $\left\{F: \operatorname{supp}\left(m_{\mathbf{u}}^{\sigma_{j}}\right) \subseteq F \subseteq \sigma_{j}\right\}$ and so $\mathbf{x}^{\mathbf{v}}=\mathbf{x}^{\mathbf{u}} \cdot \mathbf{x}^{\mathbf{v}-\mathbf{u}} \in\left(\mathbf{x}^{\mathbf{u}} \cdot m_{\mathbf{u}}^{\sigma_{j}}, \sigma_{j}\right)$. This implies that $\bigcup_{j=1}^{t}\left(\mathbf{x}^{\mathbf{u}}, \sigma_{j}\right)=\bigcup_{j=1}^{t}\left(\mathbf{x}^{\mathbf{u}} \cdot m_{\mathbf{u}}^{\sigma_{j}}, \sigma_{j}\right)$ where the inclusion $\subseteq$ follows from the previous line and $\supseteq$ from the fact that $\left(\mathbf{x}^{\mathbf{u}} \cdot m_{\mathbf{u}}^{\sigma_{j}}, \sigma_{j}\right) \subseteq\left(\mathbf{x}^{\mathbf{u}}, \sigma_{j}\right)$ for each $j=1, \ldots, t$. To see that $\bigcup_{j=1}^{t}\left(\mathbf{x}^{\mathbf{u}} \cdot m_{\mathbf{u}}^{\sigma_{j}}, \sigma_{j}\right)$ is a partition, suppose $\mathbf{x}^{\mathbf{v}} \in\left(\mathbf{x}^{\mathbf{u}} \cdot m_{\mathbf{u}}^{\sigma_{i}}, \sigma_{i}\right) \cap\left(\mathbf{x}^{\mathbf{u}} \cdot m_{\mathbf{u}}^{\sigma_{j}}, \sigma_{j}\right)$ where $i<j$. Then $\mathbf{x}^{\mathbf{v}-\mathbf{u}}$ has support in $I_{\mathbf{u}}^{\sigma_{i}} \cap I_{\mathbf{u}}^{\sigma_{j}}=\emptyset$ which implies that $\mathbf{x}^{\mathbf{v}}=\mathbf{x}^{\mathbf{u}}$. However, for $j>1$, $m_{\mathbf{u}}^{\sigma_{j}} \neq 1$ as $Q_{\mathbf{u}}^{\sigma_{j}} \neq \emptyset$ which means that $\mathbf{x}^{\mathbf{u}}$ lies only in $\left(\mathbf{x}^{\mathbf{u}}, \sigma_{1}\right)$.

Example 2.4 (continued) As before, the monomial $g$ is a root of $\mathcal{S}(J)$ with the shelling order induced on $\operatorname{star}(\delta(g))$ as above. We attain the monomials $m_{g}^{\sigma_{3}}=1$ and $m_{g}^{\sigma_{4}}=a$ which is clear from $Q_{g}^{\sigma_{3}}=\varnothing$ and $Q_{g}^{\sigma_{4}}=\{1\}$. Then $(g,\{4,11,13\}) \cup(g,\{1,4,13\})=$ $(g,\{4,11,13\}) \cup(g \cdot a,\{1,4,13\})$ with the latter union being disjoint.

Theorem 3.16 Let $\sigma_{1}, \ldots, \sigma_{s}$ be the fixed shelling of $\Delta_{\succ}$. Then

$$
\bigcup_{i=1}^{s} \bigcup_{\left(\mathbf{x}^{\mathbf{u}}, \sigma_{i}\right) \in \mathcal{S}(J)}\left(\mathbf{x}^{\mathbf{u}} \cdot m_{\mathbf{u}}^{\sigma_{i}}, \sigma_{i}\right)
$$

is a Stanley decomposition of $J$.

Proof: Lemma 3.15 showed how to make the union of the standard pairs of $\mathcal{S}(J)$ with a common root a disjoint union of pairs of $J$. By Lemma 3.6, the union (1) of these disjoint unions is a Stanley decomposition of $J$.

The above Stanley decomposition can be organized to have more structure. 
Definition 3.17 [12] Let $M \subseteq R$ be a monomial ideal. A Stanley filtration of $M$ is a Stanley decomposition of $M$ with an ordering of the pairs $\left\{\left(\mathbf{x}^{\mathbf{v}_{i}}, \tau_{i}\right): 1 \leq i \leq r\right\}$ such that for all $1 \leq j \leq r$ the set $\left\{\left(\mathbf{x}^{\mathbf{v}_{i}}, \tau_{i}\right): 1 \leq i \leq j\right\}$ is a Stanley decomposition of $M_{j}:=M+\left\langle\mathbf{x}^{\mathbf{v}_{j+1}}, \mathbf{x}^{\mathbf{v}_{j+2}}, \ldots, \mathbf{x}^{\mathbf{v}_{r}}\right\rangle$. Equivalently, the ordered set $\left\{\left(\mathbf{x}^{\mathbf{v}_{i}}, \tau_{i}\right): 1 \leq i \leq r\right\}$ is a Stanley filtration provided the modules $R / M_{j}$ form a filtration $\mathbb{K}=R / M_{0} \subsetneq R / M_{1} \subsetneq$ $R / M_{2} \subsetneq \cdots \subsetneq R / M_{r}=R / M$ with $\frac{R / M_{j}}{R / M_{j-1}} \cong \mathbb{K}\left[x_{i}: i \in \tau_{j}\right]$.

Example 3.18 (from [12]) Let $M=\left\langle x_{1} x_{2} x_{3}\right\rangle \subset \mathbb{K}\left[x_{1}, x_{2}, x_{3}\right]$. Then

$$
\left\{(1, \emptyset),\left(x_{1},\{1,2\}\right),\left(x_{2},\{2,3\}\right),\left(x_{3},\{1,3\}\right)\right\}
$$

is a Stanley decomposition of $M$ but no ordering of these pairs is a Stanley filtration of $M$. Alternatively, the ordered pairs $(1,\{1,3\}),\left(x_{2},\{2,3\}\right),\left(x_{1} x_{2},\{1,2\}\right)$ form a Stanley filtration of $M$.

We now show that the pairs in (1) can be ordered to yield a Stanley filtration of $J$. The significance of this for us comes from a result of Simon [15], interpreted as follows by Maclagan and Smith [12].

Theorem 3.19 If $M \subseteq R$ is a monomial ideal with a Stanley filtration such that for each face $\tau$ of a pair in the filtration, the prime ideal $P_{\tau}$ is a minimal prime of $M$, then $M$ is Cohen-Macaulay.

The faces of pairs in the union (1) already index minimal primes of $J$. Thus to show that $J$ is Cohen-Macaulay all we need to do is to order the pairs in (1) so that the ordered decomposition is a Stanley filtration. We do this using the following algorithm.

Algorithm 3.20 Input: The Stanley decomposition (1) of $J$.

Output: A Stanley filtration of $J$ with the same faces as those in (1).

1: (Local Lists) For each $\sigma_{i}, 1 \leq i \leq s$, order the pairs in (1) with face $\sigma_{i}$ in any way such that if $\left(\mathbf{x}^{\mathbf{u}} \cdot m_{\mathbf{u}}^{\sigma_{i}}, \sigma_{i}\right)$ precedes $\left(\mathbf{x}^{\mathbf{v}} \cdot m_{\mathbf{v}}^{\sigma_{i}}, \sigma_{i}\right)$ then $\mathbf{x}^{\mathbf{v}}$ does not divide $\mathbf{x}^{\mathbf{u}}$. Call this list $L_{i}$.

2: (Global List) The global list $\mathcal{L}$ is obtained by appending $L_{i}$ to the end of $L_{i-1}$ for $i=2, \ldots, s$.

Proof: Let $r_{i}:=\sum_{l=1}^{i} \operatorname{vol}\left(\sigma_{l}\right)$ for $i=1, \ldots, s$. Then $r:=r_{s}$ is the total number of pairs in (1). Write $\mathcal{L}$ as $\left[\left(\mathbf{x}^{\mathbf{u}_{l}} \cdot m_{\mathbf{u}}, \tau_{l}\right): 1 \leq l \leq r\right]$ where $\tau_{l}=\sigma_{i}$ when $r_{i-1}<l \leq r_{i}\left(r_{0}:=0\right)$ and $\mathbf{x}^{\mathbf{u}_{l}} \cdot m_{\mathbf{u}_{l}}^{\tau_{l}}$ is the root of the $\left(l-r_{i-1}\right)$-th pair in the local list $L_{i}$ constructed in Step 1 of the algorithm. For $1 \leq j \leq r$ define the partial list $\mathcal{L}_{j}:=\left[\left(\mathbf{x}^{\mathbf{u}_{l}} \cdot m_{\mathbf{u}_{l}}^{\tau_{l}}, \tau_{l}\right): 1 \leq l \leq j\right]$ and the ideal $M_{j}:=J+\left\langle\mathbf{x}^{\mathbf{u}_{j+1}} \cdot m_{\mathbf{u}_{j+1}}^{\tau_{j+1}}, \mathbf{x}^{\mathbf{u}_{j+2}} \cdot m_{\mathbf{u}_{j+2}}^{\tau_{j+2}}, \ldots, \mathbf{x}^{\mathbf{u}_{r}} \cdot m_{\mathbf{u}_{r}}^{\tau_{r}}\right\rangle$. We need to prove that $\mathcal{L}_{j}$ is a Stanley decomposition of $M_{j}$. Since $\mathcal{L}_{j}$ is already a partition, it suffices to show that the set of monomials in the pairs in $\mathcal{L}_{j}$ is the set of standard monomials of $M_{j}$.

(i) The standard monomials of $M_{j}$ are contained in the pairs in $\mathcal{L}_{j}$ : A standard monomial $\mathbf{x}^{\mathbf{u}}$ of $M_{j}$ is a standard monomial of $J$ and hence is covered by a unique pair $\left(\mathbf{x}^{\mathbf{u}_{l}} \cdot m_{\mathbf{u}_{l}}^{\tau_{l}}, \tau_{l}\right)$ 
in $\mathcal{L}$. Also, $\mathbf{x}^{\mathbf{u}} \notin\left\langle\mathbf{x}^{\mathbf{u}_{j+1}} \cdot m_{\mathbf{u}_{j+1}}^{\tau_{j+1}}, \ldots, \mathbf{x}^{\mathbf{u}_{r}} \cdot m_{\mathbf{u}_{r}}^{\tau_{r}}\right\rangle$ which implies that $\mathbf{x}^{\mathbf{u}} \notin\left(\mathbf{x}^{\mathbf{u}_{j+k}} \cdot m_{\mathbf{u}_{j+k}}^{\tau_{j+k}}, \tau_{j+k}\right)$ for any $k \geq 1$. Hence $l \leq j$ and $\mathbf{x}^{\mathbf{u}} \in\left(\mathbf{x}^{\mathbf{u}_{l}} \cdot m_{\mathbf{u} l}^{\tau_{l}}, \tau_{l}\right) \in \mathcal{L}_{j}$.

(ii) The monomials in the pairs in $\mathcal{L}_{j}$ are standard monomials of $M_{j}$ : Suppose $\mathbf{x}^{\mathbf{u}}$ lies in the (unique) pair $\left(\mathbf{x}^{\mathbf{u}_{l}} \cdot m_{\mathbf{u}_{l}}^{\tau_{l}}, \tau_{l}\right) \in \mathcal{L}_{j}$. Since $\mathbf{x}^{\mathbf{u}} \notin J$, it suffices to show that $\mathbf{x}^{\mathbf{u}} \notin$ $\left\langle\mathbf{x}^{\mathbf{u}_{j+1}} \cdot m_{\mathbf{u}_{j+1}}^{\tau_{j+1}}, \ldots, \mathbf{x}^{\mathbf{u}_{r}} \cdot m_{\mathbf{u}_{r}}^{\tau_{r}}\right\rangle$.

Suppose $\mathbf{x}^{\mathbf{u}} \in\left\langle\mathbf{x}^{\mathbf{u}_{j+1}} \cdot m_{\mathbf{u}_{j+1}}^{\tau_{j+1}}, \ldots, \mathbf{x}^{\mathbf{u}_{r}} \cdot m_{\mathbf{u}_{r}}^{\tau_{r}}\right\rangle$. Then there exists $p, j+1 \leq p \leq r$ such that $\mathbf{x}^{\mathbf{u}_{p}} \cdot m_{\mathbf{u}_{p}}^{\tau_{p}} \mid \mathbf{x}^{\mathbf{u}}=\mathbf{x}^{\mathbf{u}_{l}} \cdot m_{\mathbf{u}_{l}}^{\tau_{l}} \cdot \mathbf{x}_{\tau_{l}}^{*}$ where $\mathbf{x}_{\tau_{l}}^{*}$ is a monomial with support in $\tau_{l}$. Since $\operatorname{supp}\left(\mathbf{x}^{\mathbf{u}_{p}}\right)$ and $\operatorname{supp}\left(\mathbf{x}^{\mathbf{u}_{l}}\right)$ are both in $[n] \backslash\left(\tau_{p} \cup \tau_{l}\right)$, it follows that $\mathbf{x}^{\mathbf{u}_{p}} \mid \mathbf{x}^{\mathbf{u}_{l}}$. Since $l<p$, by Step 1 of the algorithm, $\tau_{p} \neq \tau_{l}$. Recall that $\left(\mathbf{x}^{\mathbf{u}_{l}}, \tau_{l}\right)$ and $\left(\mathbf{x}^{\mathbf{u}_{p}}, \tau_{p}\right)$ are standard pairs of $J$. Since $\mathbf{x}^{\mathbf{u}_{p}} \mid \mathbf{x}^{\mathbf{u}_{l}}$, by Corollary 3.5, $\left(\mathbf{x}^{\mathbf{u}_{p}}, \tau_{l}\right)$ is also in $\mathcal{S}(J)$. This implies that $\tau_{p}$ and $\tau_{l}$ are two distinct facets in $\operatorname{star}\left(\delta\left(\mathbf{x}^{\mathbf{u}_{p}}\right)\right)$. Since $m_{\mathbf{u}_{p}}^{\tau_{p}} \mid \mathbf{x}^{\mathbf{u}}, Q_{\mathbf{u}_{p}}^{\tau_{p}}\left(=\operatorname{supp}\left(m_{\mathbf{u}_{p}}^{\tau_{p}}\right)\right) \subseteq \operatorname{supp}\left(\mathbf{x}^{\mathbf{u}}\right) \cap \bigcup_{i=1}^{S} \sigma_{i} \subseteq \tau_{l}$. However, this is a contradiction since $\tau_{l}$ precedes $\tau_{p}$ in the shelling order on $\Delta_{\succ}$ and hence $Q_{\mathbf{u}_{p}}^{\tau_{p}}$ cannot be in $\tau_{l}$. Thus $m_{\mathbf{u}_{p}}^{\tau_{p}} \not \mathbf{x}^{\mathbf{u}}$ and $\mathbf{x}^{\mathbf{u}} \notin\left\langle\mathbf{x}^{\mathbf{u}_{j+1}} \cdot m_{\mathbf{u}_{j+1}}^{\tau_{j+1}}, \ldots, x^{\mathbf{u}_{r}} \cdot m_{\mathbf{u}_{r}}^{\tau_{r}}\right\rangle$ and thus not in $M_{j}$.

Example 2.4 (continued) As before, $\sigma_{1}=\{4,11,12\}, \sigma_{2}=\{11,12,13\}, \sigma_{3}=$ $\{4,11,13\}$ and $\sigma_{4}=\{1,4,13\}$ is a shelling order on $\Delta_{\succ}$. The (ordered) local lists in the Stanley filtration produced by Algorithm 3.20 are:

$\mathcal{L}_{1}=[(1,\{4,11,12\}),(h,\{4,11,12\})]$,

$\mathcal{L}_{2}=[(1 \cdot m,\{11,12,13\})]$,

$\mathcal{L}_{3}=[(1 \cdot d m,\{4,11,13\}),(g,\{4,11,13\}),(j,\{4,11,13\})]$,

$\mathcal{L}_{4}=[(1 \cdot a,\{1,4,13\}),(b,\{1,4,13\}),(c,\{1,4,13\}),(e,\{1,4,13\}),(f,\{1,4,13\})$,

$(g \cdot a,\{1,4,13\}),(i,\{1,4,13\}),(j \cdot a,\{1,4,13\}),(b j,\{1,4,13\})]$.

Proof of Theorem 3.1: Algorithm 3.2 shows that the initial ideal $J$ of Theorem 2.3 has a Stanley filtration that satisfies the conditions of Theorem 3.19. This theorem guarantees that $J$ is Cohen-Macaulay.

Remark 3.21 We remark that even when $\mathcal{A}$ is $\Delta$-normal it is not true that all initial ideals of $I_{\mathcal{A}}$ without embedded primes are Cohen-Macaulay. Take $\mathcal{A}$ to be the columns of

$$
\mathbf{A}=\left(\begin{array}{llllllll}
1 & 1 & 1 & 1 & 1 & 1 & 1 & 1 \\
2 & 2 & 1 & 0 & 1 & 1 & 1 & 1 \\
0 & 1 & 2 & 1 & 2 & 1 & 2 & 1 \\
1 & 0 & 2 & 0 & 0 & 0 & 1 & 1
\end{array}\right)
$$

Then $\mathcal{A}$ admits a unimodular regular triangulation and is hence $\Delta$-normal. The toric ideal $I_{\mathcal{A}} \subset \mathbb{K}[a, \ldots, h]$ has codimension four and has 46 initial ideals without embedded primes. Among them, the following two have projective dimension five.

(1) $\left\langle a c d, a d g, a f g, a e, a g^{2}, c e, c f, e h, f^{2}, b c^{2} d, f g h\right\rangle$

(2) $\left\langle a c d, a d g, a f g, a e, a g^{2}, c e, c f, e h, f^{2}, f g h, g^{2} h^{2}\right\rangle$ 
The initial ideals of $I_{\mathcal{A}}$ were computed using the software package CaTS [6] and then checked for embedded primes and Cohen-Macaulayness using Macaulay 2. This example was found by systematic computer search. The matrix presented above, suggested by the referee, is a nicer row equivalent matrix to the matrix we found. We remark that the first example of a monomial toric initial ideal without embedded primes that is not CohenMacaulay was found by Laura Matusevich [13]. In that example, $I_{\mathcal{A}}$ is not Cohen-Macaulay and thus $\mathcal{A}$ is not normal.

\section{Degree bounds}

Theorem 4.1 If $\mathcal{A}$ is a graded $\Delta$-normal configuration, then there exists a term order $\succ$ such that $\Delta=\Delta_{\succ}$ and the Gröbner basis of $I_{\mathcal{A}}$ with respect to $\succ$ consists of binomials of degree at most $d=\operatorname{dim}(\mathcal{A})$.

Theorem 4.1 settles Conjecture 1.2 for the subset of normal configurations that are $\Delta$ normal. Since $\mathcal{A}$ is graded, $I_{\mathcal{A}}$ is homogeneous with respect to the usual grading of $R$ where $\operatorname{deg}\left(x_{i}\right)=1$ for $i=1, \ldots, n$. Hence it suffices to show that $I_{\mathcal{A}}$ has an initial ideal of degree at most $d$. We will show that the initial ideal $J$ from Theorem 2.3 satisfies this degree bound when $\mathcal{A}$ is graded. This is done by classifying the generators of $J$ into three types, each of which have degree at most $d$. The classification arises naturally via the projection maps $\left\{\pi_{\sigma}: \sigma \in \max \Delta_{\succ}\right\}$ defined in Section 3.

Proposition 13.15 in [17] shows that Conjecture 1.2 is true whenever $\mathcal{A}$ admits a regular unimodular triangulation. (See also Proposition 13.18 in [17].) Such configurations form a proper subset of the set of $\Delta$-normal configurations. For any configuration $\mathcal{A}$ and positive scalar $c$ we can define $c \cdot \mathcal{A}$ as the configuration given by multiplying each point in $\mathcal{A}$ by the scalar $c$. Then for every graded normal $\mathcal{A}$ there exists a positive integer $c_{\mathcal{A}}$ such that the configuration defined by all the lattice points in the convex hull of $c_{\mathcal{A}} \cdot \mathcal{A}$ admits regular unimodular triangulations and thus has a Gröbner basis of degree at most $d$. See [1] and [2] for many such results.

Note that Conjecture 1.2 requires that $\mathcal{A}$ be both graded and normal.

Example 4.2 Graded, but not normal: When $\mathcal{A}=\{(1,0),(1, p),(1, q)\}$ with $0<p<q$, $q>2$ and $g . c . d(p, q)=1$, then $I_{\mathcal{A}}=\left\langle x_{1}^{q-p} x_{3}^{p}-x_{2}^{q}\right\rangle$. Its two initial ideals are therefore generated in degree $q>2=d$.

Normal, but not graded: The normal configuration $\mathcal{A}=\{(1,0),(1,1),(p, p+1)\}$ where $p \geq 2$ has the toric ideal $I_{\mathcal{A}}=\left\langle x_{1} x_{3}-x_{2}^{p+1}\right\rangle$. Hence $x_{1} x_{3}-x_{2}^{p+1}$ is the unique element in both its reduced Gröbner bases.

Remark 4.3 ( [17], Chapter 13) The bound in Conjecture 1.2 is best possible. Consider the graded $\Delta$-normal configuration $\mathcal{A}=\left\{d \mathbf{e}_{1}, d \mathbf{e}_{2}, \ldots, d \mathbf{e}_{d}, \mathbf{e}_{1}+\mathbf{e}_{2}+\cdots+\mathbf{e}_{d}\right\}$ where $d \in \mathbb{N}$. (Note that cone $(\mathcal{A})$ is simplicial). Then $I_{\mathcal{A}}=\left\langle x_{1} x_{2} \cdots x_{d}-x_{d+1}^{d}\right\rangle$.

Consider the initial ideal $J$ from Theorem 2.3 for a graded $\Delta_{\succ}$-normal $\mathcal{A}$. Since $\mathcal{A}$ is graded, we may assume without loss of generality that $\mathbf{a}_{i}=\left(1, \mathbf{a}_{i}^{\prime}\right) \in \mathbb{Z}^{d}$ for $i=1, \ldots, n$. We will show that $J$ is generated in degree at most $d$. 
For a $\sigma \in \max \Delta_{\succ}$, recall that $\sigma_{\text {in }}:=\left\{i: \mathbf{a}_{i} \in \operatorname{cone}\left(\mathcal{A}_{\sigma}\right), i \notin \sigma\right\}$. Define $\sigma_{\text {out }}:=$ $\left\{i: \mathbf{a}_{i} \notin \operatorname{cone}\left(\mathcal{A}_{\sigma}\right)\right\}$. Then $\sigma \cup \sigma_{\text {in }} \cup \sigma_{\text {out }}$ is a partition of $[n]$. Let $J^{\sigma}:=\pi_{\sigma}(J)$ be the artinian ideal in $R_{\sigma}=\mathbb{K}\left[x_{j}: j \in \sigma_{\text {in }} \cup \sigma_{\text {out }}\right]$ from Theorem 3.4. Recall that the standard monomials of $J^{\sigma}$ are the roots of standard pairs in $\mathcal{S}(J)$ with face $\sigma$. Since the supports of these roots lie in $\sigma_{\text {in }}, J^{\sigma} \cap \mathbb{K}\left[x_{i}: i \in \sigma_{\text {in }}\right]$ is a monomial ideal $N^{\sigma}=\left\langle\mathbf{x}^{\mathbf{v}_{1}}, \mathbf{x}^{\mathbf{v}_{2}}, \ldots, \mathbf{x}^{\mathbf{v}_{t \sigma}}\right\rangle$ with $\operatorname{supp}\left(\mathbf{x}^{\mathbf{v}_{i}}\right) \subseteq \sigma_{\text {in }}$, and

$$
J^{\sigma}=\left\langle x_{j}: j \in \sigma_{\text {out }}\right\rangle+N^{\sigma}
$$

Lemma 4.4 Each minimal generator $\mathbf{x}^{\mathbf{v}_{i}}$ of $N^{\sigma}$ is a minimal generator of $J$ of degree at most $d$.

Proof: A minimal generator $\mathbf{x}^{\mathbf{v}_{i}}$ of $N^{\sigma}$ is the projection via $\pi_{\sigma}$ of a minimal generator $\mathbf{x}^{\mathbf{v}_{i}} \mathbf{x}_{\sigma}^{\mathbf{m}}$ of $J$ where $\operatorname{supp}\left(\mathbf{x}_{\sigma}^{\mathbf{m}}\right) \subseteq \sigma$. Suppose $\operatorname{supp}\left(\mathbf{x}_{\sigma}^{\mathbf{m}}\right) \neq \emptyset$. Then $\mathbf{x}^{\mathbf{v}_{i}}$ is a standard monomial of $J$ with $\operatorname{supp}\left(\mathbf{x}^{\mathbf{v}_{i}}\right) \subseteq \sigma_{\text {in }}$. Hence $\mathbf{x}^{\mathbf{v}_{i}}$ is covered by a standard pair $\left(\mathbf{x}^{\mathbf{u}_{\gamma}}, \sigma\right)$ of $J$. This implies that all monomials of the form $\mathbf{x}^{\mathbf{v}_{i}} \mathbf{x}_{\sigma}^{\mathbf{p}}$ as $\mathbf{p}$ varies are standard monomials of $J$ which contradicts that $\mathbf{x}^{\mathbf{v}_{i}} \mathbf{x}_{\sigma}^{\mathbf{m}}$ is in $J$. Thus $\operatorname{supp}\left(\mathbf{x}_{\sigma}^{\mathbf{m}}\right)=\emptyset$ which implies that $\mathbf{x}^{\mathbf{v}_{i}}$ is a minimal generator of $J$.

Since $\mathbf{a}_{i}=\left(1, \mathbf{a}_{i}^{\prime}\right) \in \mathbb{Z}^{d}$ for $i \in[n]$, each lattice point in the half open fundamental parallelopiped $\mathrm{FP}_{\sigma}$ of cone $\left(\mathcal{A}_{\sigma}\right)$ lies on one of the $d$ hyperplanes $x_{1}=0, \ldots, x_{1}=d-1$ in $\mathbb{R}^{d}$. Therefore, if $\gamma \in \mathrm{FP}_{\sigma} \cap \mathbb{Z}^{d}$, then the 1-norm of $\mathbf{u}_{\gamma}$ which equals the first co-ordinate of $\left(\mathbf{A} \mathbf{u}_{\gamma}\right)$ which equals $\gamma_{1}$ is at most $d-1$. This implies that $\operatorname{deg}\left(\mathbf{x}^{\mathbf{u}_{\gamma}}\right) \leq d-1$. Thus all standard monomials of the artinian ideal $J^{\sigma}$ have degree at most $d-1$ which implies that the minimal generators of $J^{\sigma}$ (and $N^{\sigma}$ ) have degree at most $d$.

Example 2.4 (continued) For $\sigma=\{1,4,13\}, J^{\sigma}=\langle h, k, l\rangle+\left(N^{\sigma}=\left\langle j^{2}, g j, i j, f j, i g\right.\right.$, $\left.\left.g^{2}, c g, e j, i^{2}, f i, c^{2}, f^{2}, c i, e g, f g, c j, c f, b g, e i, b i, e f, b f, e c, b c, e^{2}, b e, b^{2}\right\rangle\right)$. Note that all minimal generators of $N^{\sigma}$ are minimal generators of $J$ of degree at most three.

Theorem 4.5 If $\mathcal{A}$ is a graded normal configuration with cone $(\mathcal{A})$ simplicial then $I_{\mathcal{A}}$ has a Gröbner basis consisting of binomials of degree at most $d$.

Proof: Assuming that cone $(\mathcal{A})$ is generated by $\mathbf{a}_{1}, \ldots, \mathbf{a}_{d}, \mathcal{A}$ is $\Delta_{\succ}$-normal where $\Delta_{\succ}$ is the regular triangulation of $\mathcal{A}$ with the unique facet $\sigma=[d]$. Here $\sigma_{\text {out }}=\emptyset$.

We argue that all minimal generators of $J$ have support in $\sigma_{\text {in }}=\{d+1, \ldots, n\}$. Suppose $\mathbf{x}^{\alpha}$ is a minimal generator of $J$ with $\operatorname{supp}\left(\mathbf{x}^{\alpha}\right) \cap[d]=F \neq \emptyset$. Let $G=\operatorname{supp}\left(\mathbf{x}^{\alpha}\right) \backslash[d]$. Then $G \neq \emptyset$ since otherwise $\mathbf{x}^{\alpha}$ would lie on the standard pair $(1,[d])$ of $J$ which is a contradiction. Write $\mathbf{x}^{\alpha}=\mathbf{x}^{\alpha_{F}} \mathbf{x}^{\alpha_{G}}$ where $\operatorname{supp}\left(\alpha_{F}\right) \subseteq F$ and $\operatorname{supp}\left(\alpha_{G}\right) \subseteq G$. Since $G, F \neq \emptyset, \mathbf{x}^{\alpha_{G}}$ is a standard monomial of $J$ which implies that $\mathbf{x}^{\alpha}$ is also a standard monomial of $J$ as $\mathbf{x}^{\alpha_{G}}$ lies on some standard pair with face $[d]$. This is a contradiction and so $F=\emptyset$.

The above argument shows that $J$ and $N^{\sigma}$ have the same minimal generators. The degree bound then follows from the proof of Lemma 4.4. 
Theorem 4.5 proves Theorem 4.1 in the case where cone $(\mathcal{A})$ is simplicial. When cone $(\mathcal{A})$ is not simplicial, $J$ may have minimal generators that are not pre-images under $\pi_{\sigma}$ of the minimal generators of $N^{\sigma}$ (or even $J^{\sigma}$ ) as $\sigma$ varies in max $\Delta_{\succ}$. Our next step is to show that for a $\sigma \in \max \Delta_{\succ}$, the minimal generators of $J$ that project under $\pi_{\sigma}$ to the minimal generators $x_{j} \in \sigma_{\text {out }}$ of $J^{\sigma}$ have degree at most $d$. We need a preliminary lemma.

Let $Q$ be a $(d-1)$-polytope in $\left\{\mathbf{x} \in \mathbb{R}^{d}: x_{1}=1\right\}$ and let $C$ be the cone over $Q$. Then there exists a matrix $\mathbf{S} \in \mathbb{R}^{f \times d}$ such that $C=\left\{\mathbf{x} \in \mathbb{R}^{d}: \mathbf{S x} \geq 0\right\}$ where each row of $\mathbf{S}$ is the normal to a facet of $C$. Hence $Q=\left\{\mathbf{x} \in \mathbb{R}^{d}: x_{1}=1\right.$, Sx $\left.\geq \mathbf{0}\right\}$. Let $Q_{\text {rev }}$ be the system obtained by reversing all the inequalities in $Q$ :

$$
Q_{\mathrm{rev}}=\left\{\mathbf{x} \in \mathbb{R}^{d}: x_{1}=1, \mathbf{S x} \leq \mathbf{0}\right\} .
$$

Lemma 4.6 The polyhedron defined by $Q_{\mathrm{rev}}$ is the empty set.

Proof: We may assume that $Q$ has been translated so that the unit vector $\mathbf{e}_{1} \in \mathbb{R}^{d}$ lies in the relative interior of $Q$. If $\mathbf{x} \in C$ then by our assumption, $x_{1} \geq 0$ which implies that $\mathbf{e}_{1} \cdot \mathbf{x}\left(=x_{1}\right) \geq 0$. This implies that $\mathbf{e}_{1} \in C^{*}=\{\mathbf{y S}: \mathbf{y} \geq \mathbf{0}\}$ where $C^{*}$ is the dual cone to $C$. (Recall $C^{*}:=\left\{\mathbf{v} \in \mathbb{R}^{d}: \mathbf{v} \cdot \mathbf{x} \geq 0\right.$, for all $\left.\mathbf{x} \in C\right\}$.) Thus there exists some $\mathbf{y} \geq \mathbf{0}, \mathbf{y} \neq \mathbf{0}$ such that $\mathbf{y S}=\mathbf{e}_{1}$. Therefore, if we choose $\mathbf{v} \in \mathbb{R}^{2+f}$ such that $\mathbf{v}=(0,1, \mathbf{y})$ then $\mathbf{v} \geq \mathbf{0}$, $\mathbf{v} \neq \mathbf{0}$ and

$$
\mathbf{v} \cdot\left(\begin{array}{cccc}
1 & 0 & \cdots & 0 \\
-1 & 0 & \cdots & 0 \\
s_{11} & s_{12} & \cdots & s_{1 d} \\
\vdots & \vdots & \vdots & \vdots \\
s_{f 1} & s_{f 2} & \cdots & s_{f d}
\end{array}\right)=0
$$

Let $z=(1,-1,0, \ldots, 0)$ be the right hand side vector in the description of $Q_{\text {rev }}$ by inequalities. Then $\mathbf{v} \cdot \mathbf{z}=1(-1)=-1<0$ and by Farkas' lemma [20, Proposition 1.7.], $Q_{\text {rev }}=\emptyset$.

Lemma 4.7 Let $\sigma$ be a facet of $\Delta_{\succ}$. Then for $j \in \sigma_{\text {out }}$, the minimal generators of $J$ that are preimages of the minimal generator $x_{j}$ of $J^{\sigma}$ under the map $\pi_{\sigma}$ are squarefree monomials of degree at most $d$.

Proof: Let $\sigma \in \max \Delta_{\succ}, j \in \sigma_{\text {out }}$ and $P:=x_{j} \mathbf{x}_{\sigma}^{\mathbf{m}}$ be a minimal generator of $J$ with $Y:=\operatorname{supp}\left(\mathbf{x}_{\sigma}^{\mathbf{m}}\right) \subseteq \sigma$. All minimal generators of $J$ that project to $x_{j}$ under $\pi_{\sigma}$ look like $P$. If $Y=\emptyset$, then $x_{j}$ is the only minimal generator of $J$ that projects to $x_{j}$ and we are done. Therefore, we consider the case where $Y \neq \emptyset$.

Suppose $P$ is not squarefree. Then there exists an $i \in \sigma$ such that $m_{i}>1$ where $m_{i}$ is the $i$-th co-ordinate of $\mathbf{m}$. Since $P$ is a minimal generator of $J, P / x_{i}$ is a standard monomial of $J$ with $\operatorname{supp}\left(P / x_{i}\right)=\operatorname{supp}(P)=\{j\} \cup Y$. Hence there exists $\tau \in \max \Delta_{\succ}$ such that a standard pair with face $\tau$ covers $P / x_{i}$. This implies that $\operatorname{supp}\left(P / x_{i}\right)=\{j\} \cup Y \subseteq \tau_{\text {in }} \cup \tau$. 
Since $Y \subseteq \sigma, Y \cap \tau_{\text {in }}=\emptyset$ and thus, $Y \subseteq \tau \cap \sigma$. If $j \in \tau$, then $P$ is covered by the standard pair $(1, \tau)$ which contradicts that $P$ is in $J$. If $j \in \tau_{\text {in }}$, then $\mathbf{a}_{j}$ lies in cone $\left(\mathcal{A}_{\tau}\right)$. Since $\mathcal{A}$ lies on the hyperplane $\left\{\mathbf{x} \in \mathbb{R}^{d}: x_{1}=1\right\}, \mathbf{a}_{j}$ is in fact in the minimal Hilbert basis of both cone $\left(\mathcal{A}_{\tau}\right)$ and cone $(\mathcal{A})$ and hence $\mathbf{x}=\mathbf{e}_{j}$ is the unique vector in $\mathbb{N}^{n}$ that satisfies $\mathbf{A x}=\mathbf{a}_{j}$. Consequently $\left(x_{j}, \tau\right)$ is a standard pair of $J$. But this implies that $P$ lies on this standard pair which is again a contradiction. Therefore, $P$ is squarefree.

To argue that $\operatorname{deg}(P) \leq d$, it therefore suffices to prove that $Y \subsetneq \sigma$. Suppose $\sigma=[d]$, $\mathbf{x}_{[d]}:=\prod_{i \in \sigma} x_{i}$ and $P=x_{j} \mathbf{x}_{[d]}$. Then for each $i \in[d], P / x_{i}$ is a standard monomial of $J$ and is therefore covered by a standard pair $\left(*, \tau^{i}\right)$ of $J$. The face $\tau^{i}$ does not contain $i$ since otherwise $P$ would be a standard monomial of $J$. In particular, $\tau^{i} \neq[d]$ for any $i \in[d]$. Also, $j \in \tau^{i} \cup \tau_{\text {in }}^{i}$ for each $i \in[d]$.

We now show that we may assume $\tau^{i} \cap[d]=[d] \backslash\{i\}$ for all $i \in[d]$. Clearly, $\tau^{i} \cap[d] \subseteq$ $[d] \backslash\{i\}$ since $i \notin \tau^{i}$. Suppose a monomial in $P / x_{i} \cdot \mathbb{K}\left[x_{l}: l \in[d] \backslash\{i\}\right]$ lies in $J$. Then it is divisible by a minimal generator of $J$ that projects to $x_{j}$ under $\pi_{\sigma}$, all of which are squarefree. Such a minimal generator would properly divide $P$ which contradicts that $P$ is a minimal generator of $J$. Hence $\left(P / x_{i},[d] \backslash\{i\}\right)$ is a pair of $J$ and therefore, contained in a standard pair of $J$. We may assume that $\tau^{i}$ is the face of this standard pair. Thus $[d] \backslash\{i\} \subset \tau^{i}$ and $\tau^{i} \cap[d]=[d] \backslash\{i\}$ as claimed.

Since $\mathcal{A}$ is graded, $\tau^{1}, \ldots, \tau^{d}$ index $(d-1)$-simplices in a regular triangulation of $\operatorname{conv}(\mathcal{A})$, the convex hull of $\mathcal{A}$. The simplex indexed by $[d]$ is geometrically $Q[d]=\{\mathbf{x} \in$ $\left.\mathbb{R}^{d}: \mathbf{s}_{i} \cdot \mathbf{x} \geq 0, x_{1}=1, i=1,2, \ldots, d\right\}$ where $\mathbf{s}_{i} \cdot \mathbf{a}_{l}=0$ for all $l \in[d] \backslash\{i\}$ and $\mathbf{s}_{i} \cdot \mathbf{a}_{i}>0$. Now $j \in \tau^{i} \cup \tau_{\text {in }}^{i} \cap \sigma_{\text {out }}$ for each $i \in[d]$ implies that $\mathbf{a}_{j} \in Q[d]_{\text {rev }}$ where $Q[d]_{\mathrm{rev}}=\left\{\mathbf{x} \in \mathbb{R}^{d}: \mathbf{s}_{i} \cdot \mathbf{x} \leq 0, x_{1}=1, i=1,2, \ldots, d\right\}$. But by Lemma 4.6, $Q[d]_{\mathrm{rev}}=\emptyset$ which creates a contradiction. Therefore, $x_{j} \mathbf{x}_{[d]}$ is not a minimal generator of $J$ and all preimages $P$ of $x_{j}$ have degree at most $d$.

Example 2.4 (continued) For $\sigma=\{1,4,13\}, \sigma_{\text {out }}=\{8,11,12\}$ which index the variables $h, k, l$. The minimal generators of $J$ that map to these variables under $\pi_{\sigma}$ are $h m, a k, a l, h a$, $d m l$.

Finally we consider the minimal generators of $J$ that do not project under $\pi_{\sigma}$ to minimal generators of $J^{\sigma}$ for any $\sigma \in \max \Delta_{\succ}$. Such generators may exist.

Example 2.4 (continued) Consider the minimal generator $g h$ of $J$. Then $g h=$ $\pi_{\{1,4,13\}}(g h)=\pi_{\{4,11,13\}}(g h)=\pi_{\{4,11,12\}}(g h)=\pi_{\{11,12,13\}}(g h)$ is not a minimal generator of $J^{\sigma}$ for any of the four facets $\sigma$ of $\Delta_{\succ}$.

Lemma 4.8 Let $\mathbf{x}^{\mathbf{m}}$ be a minimal generator of $J$ whose image under $\pi_{\sigma}$ is not a minimal generator of $J^{\sigma}$ for any facet $\sigma$ of $\Delta_{\succ}$. Then $\mathbf{x}^{\mathbf{m}}$ is a quadratic squarefree monomial.

Proof: Let $\tau$ and $\tau^{\prime}$ be facets of $\Delta_{\succ}$ and let $i \in \tau_{\text {in }}, j \in \tau_{\text {in }}^{\prime}$ with $i, j \notin \tau_{\text {in }} \cap \tau_{\text {in }}^{\prime}$. Then $x_{i} x_{j}$ is not covered by any standard pair of $J$ and hence lies in $J$. Since $\mathcal{A}$ is graded, $\left(x_{i}, \tau\right)$ and $\left(x_{j}, \tau^{\prime}\right)$ are standard pairs of $J$ which implies that $x_{i} x_{j}$ is a minimal generator of $J$. Further, $\pi_{\sigma}\left(x_{i} x_{j}\right)$ is not a minimal generator of $J^{\sigma}$ for any $\sigma \in \max \Delta_{\succ}$. We will prove that $L:=\left\{x_{i} x_{j}: i \in \tau_{\text {in }}, j \in \tau_{\text {in }}^{\prime}\right.$ and $\left.i, j \notin \tau_{\text {in }} \cap \tau_{\text {in }}^{\prime}\right\}$ is precisely the set of minimal generators 
of $J$ that do not project under $\pi_{\sigma}$ to a minimal generator of $J^{\sigma}$ for a $\sigma \in \max \Delta_{\succ}$. This will prove the lemma.

Suppose $\mathbf{x}^{\mathbf{m}}$ is a minimal generator of $J$ such that $\pi_{\sigma}\left(\mathbf{x}^{\mathbf{m}}\right)$ is not a minimal generator of $J^{\sigma}$ for any $\sigma \in \max \Delta_{\succ}$. Let $Y:=\operatorname{supp}\left(\mathbf{x}^{\mathbf{m}}\right)$.

Case (i) $Y \subseteq \sigma_{\text {in }}$ for some $\sigma \in \max \Delta_{\succ}$ : Then $\mathbf{x}^{\mathbf{m}} \in N^{\sigma}=J^{\sigma} \cap \mathbb{K}\left[x_{j}: j \in \sigma_{\text {in }}\right]$. Since $\mathbf{x}^{\mathbf{m}}$ is not a minimal generator of $J^{\sigma}$ (and hence $N^{\sigma}$ ), some minimal generator of $N^{\sigma}$ properly divides $\mathbf{x}^{\mathbf{m}}$. By Lemma 4.4, every minimal generator of $N^{\sigma}$ is a minimal generator of $J$ which contradicts that $\mathbf{x}^{\mathbf{m}}$ is a minimal generator of $J$.

Case (ii) $Y \subseteq \sigma$ for some $\sigma \in \max \Delta_{\succ}$ : Then $\mathbf{x}^{\mathbf{m}}$ is covered by the standard pair $(1, \sigma)$ which contradicts that $\mathbf{x}^{\mathbf{m}} \in J$.

Case (iii) $Y \subseteq \sigma \cup \sigma_{\text {in }}$ for some $\sigma \in \max \Delta_{\succ}$, with $Y \cap \sigma \neq \emptyset$ and $Y \cap \sigma_{\text {in }} \neq \emptyset$ : Write $\mathbf{x}^{\mathbf{m}}=\mathbf{x}^{\mathbf{m}^{\prime}} \mathbf{x}^{\mathbf{m}^{\prime \prime}}$ where $\emptyset \neq \operatorname{supp}\left(\mathbf{x}^{\mathbf{m}^{\prime}}\right) \subseteq \sigma$ and $\emptyset \neq \operatorname{supp}\left(\mathbf{x}^{\mathbf{m}^{\prime \prime}}\right) \subseteq \sigma_{\text {in }}$. Then $\mathbf{x}^{\mathbf{m}^{\prime \prime}} \in N^{\sigma}$ all of whose minimal generators are minimal generators of $J$. This implies that a divisor of $\mathbf{x}^{\mathbf{m}^{\prime \prime}}$ is a minimal generator of $J$. Therefore, $\mathbf{x}^{\mathbf{m}}$ is not a minimal generator of $J$, a contradiction.

The above cases have shown that there is no single $\sigma \in \max \Delta_{\succ}$ such that $Y \subseteq \sigma \cup \sigma_{\text {in }}$. Therefore, there exist two distinct $\sigma, \tau \in \max \Delta_{\succ}$ and two indices $i, j \in Y$ such that $i \in \sigma \cup \sigma_{\text {in }} \cap \tau_{\text {out }}$ and $j \in \tau \cup \tau_{\text {in }} \cap \sigma_{\text {out }}$.

Case (a) $i \in \sigma$ : Since $j \in \sigma_{\text {out }}, x_{i} x_{j}$ is not covered by any standard pair of $J$ and so lies in $J$. Since $x_{i} x_{j}$ divides $\mathbf{x}^{\mathbf{m}}$ and $\mathbf{x}^{\mathbf{m}}$ is a minimal generator of $J$ it must be that $\mathbf{x}^{\mathbf{m}}=x_{i} x_{j}$. But then $\pi_{\sigma}\left(\mathbf{x}^{\mathbf{m}}\right)=x_{j}, j \in \sigma_{\text {out }}$ is a minimal generator of $J^{\sigma}$ which contradicts our choice of $\mathbf{x}^{\mathbf{m}}$. Therefore this case cannot arise.

Case (b) $j \in \tau$ : By a symmetric argument to the previous, this cannot happen.

Therefore, the only possibility is that $i \in \sigma_{\text {in }}$ and $j \in \tau_{\text {in. }}$. Since $i \in \tau_{\text {out }}$ and $j \in \sigma_{\text {out }}$, $i, j \notin \sigma_{\text {in }} \cap \tau_{\text {in }}$. By the argument in the beginning of the proof, $x_{i} x_{j}$ is a minimal generator of $J$ and so $\mathbf{x}^{\mathbf{m}}=x_{i} x_{j}$. Thus $\mathbf{x}^{\mathbf{m}}$ lies in the set $L$ as claimed.

Proof of Theorem 4.1: Lemmas 4.4, 4.7 and 4.8 account for all minimal generators of the initial ideal $J$ and show that they all have degree at most $d$. Since $\mathcal{A}$ is graded, the reduced Gröbner basis of $I_{\mathcal{A}}$ with initial ideal $J$ consists of homogeneous binomials. Hence these binomials have degree at most $d$.

\section{5. $\Delta$-normal and non- $\Delta$-normal families}

In this last section we construct non-trivial families of both $\Delta$-normal and non- $\Delta$-normal configurations. Recall that any configuration $\mathcal{A}$ is always $\Delta$-normal with respect to all its regular unimodular triangulations $\Delta$. Also, a configuration $\mathcal{A}=\left\{\mathbf{a}_{1}, \ldots, \mathbf{a}_{n}\right\} \subset \mathbb{Z}^{d}$ for which cone $(\mathcal{A})$ is simplicial is $\Delta$-normal with respect to its coarsest (regular) triangulation $\Delta=\{\{1, \ldots, d\}\}$ if we assume that $\operatorname{cone}(\mathcal{A})=\operatorname{cone}\left(\left\{\mathbf{a}_{1}, \ldots, \mathbf{a}_{d}\right\}\right)$. Call $\mathcal{A}$ simplicial if cone $(\mathcal{A})$ is simplicial. We construct families of $\Delta$-normal configurations that are not simplicial and do not admit regular unimodular triangulations. By computer search, Firla and Ziegler [4] found hundreds of normal simplicial configurations $\mathcal{A}$ in $\mathbb{N}^{4}$ and $\mathbb{N}^{5}$ (in the 
course of writing [5]) that admit no unimodular triangulations. Our first result in this section is a construction that extends a Firla-Ziegler configuration to a family of non-simplicial $\Delta$ normal configurations-one in $\mathbb{Z}^{d}$ for each $d \geq 5$-without unimodular triangulations. This is based on successive suspensions of a certain triangulation of Firla-Ziegler configurations. In the second part of this section we construct a family of normal configurations (an $\mathcal{A} \subset \mathbb{Z}^{d}$ for each $d \geq 10$ ) that are not $\Delta$-normal for any regular triangulation $\Delta$. This is also done by taking successive suspensions starting with a configuration discovered by Hibi and Ohsugi in [8].

\section{1. $\Delta$-normal families from Firla-Ziegler configurations}

Each Firla-Ziegler normal simplicial $\mathcal{A} \subset \mathbb{N}^{4}$ without unimodular triangulations is the Hilbert basis of the cone generated by $\mathbf{e}_{1}, \mathbf{e}_{2}, \mathbf{e}_{3}$, the first three unit vectors in $\mathbb{R}^{4}$, and a vector $\mathbf{v} \in \mathbb{N}^{4}$ of the form $\mathbf{v}:=(a, b, c, d)^{t}$ with $0<a<b<c<d$. In this subsection we let $\mathcal{A}$ denote such a Firla-Ziegler configuration and let $\mathcal{A}_{\text {ext }}=\left\{\mathbf{e}_{1}, \mathbf{e}_{2}, \mathbf{e}_{3}, \mathbf{v}\right\}$ be the extreme rays of cone $(\mathcal{A})$. By definition, $\mathcal{A}$ is the unique minimal generating set of the semigroup $\operatorname{cone}\left(\mathcal{A}_{\text {ext }}\right) \cap \mathbb{Z}^{4}$, and cone $(\mathcal{A}) \subset \mathbb{R}_{\geq 0}^{4}$. We construct an infinite family of $\Delta$-normal, non simplicial configurations with no unimodular triangulations, starting with a Firla-Ziegler $\mathcal{A}$.

Lemma 5.1 The vector $\mathbf{1}:=(1,1,1,1)^{t}$ is contained in $\mathcal{A}$.

Proof: $\quad$ Since $\mathbf{1}=\frac{1}{d} \mathbf{v}+\frac{d-c}{d} \mathbf{e}_{3}+\frac{d-b}{d} \mathbf{e}_{2}+\frac{d-a}{d} \mathbf{e}_{1}$ and $a<b<c<d, \mathbf{1} \in \operatorname{cone}\left(\mathcal{A}_{\text {ext }}\right) \cap \mathbb{Z}^{4}=$ $\mathbb{N} \mathcal{A}$. For every $\mathbf{p}=\left(p_{1}, p_{2}, p_{3}, p_{4}\right) \in \operatorname{cone}\left(\mathcal{A}_{\text {ext }}\right)=\operatorname{cone}(\mathcal{A})$ with $p_{4}>0, p_{i}>0$ for $i=1, \ldots, 4$ since the $\mathbb{R}_{\geq 0}$-linear combination of elements in $\mathcal{A}_{\text {ext }}$ that expresses $\mathbf{p}$ as an element of cone $\left(\mathcal{A}_{\text {ext }}\right)$ must involve a positive multiple of $\mathbf{v}$. On the other hand, the $\mathbb{N}$-linear combination of elements in $\mathcal{A}$ that expresses $\mathbf{1}$ as an element of $\mathbb{N} \mathcal{A}$ is the sum of distinct vectors in $\mathcal{A} \cap\{0,1\}^{4}$. At least one of these $0-1$ vectors - say $\mathbf{w}$ - has a positive last co-ordinate which implies that $\mathbf{w}=\mathbf{1}$. Therefore, $\mathbf{1}$ is in $\mathcal{A}$, the minimal Hilbert basis of $\operatorname{cone}\left(\mathcal{A}_{\text {ext }}\right) \cap \mathbb{Z}^{4}$.

Example 5.2 The first Firla-Ziegler $\mathcal{A}$ in $\mathbb{N}^{4}$ has $\mathbf{v}=(1,2,3,5)$ and $\mathcal{A}$ consists of the columns of the matrix

$$
\mathbf{A}=\left(\begin{array}{llllllll}
1 & 0 & 0 & 1 & 1 & 1 & 1 & 1 \\
0 & 1 & 0 & 2 & 1 & 1 & 2 & 2 \\
0 & 0 & 1 & 3 & 1 & 2 & 2 & 3 \\
0 & 0 & 0 & 5 & 1 & 2 & 3 & 4
\end{array}\right)
$$

The Hilbert basis of any rational polyhedral cone can be computed using the software package Normaliz [3].

From a Firla-Ziegler $\mathcal{A}$ we will now recursively construct configurations $\mathcal{A}^{d}$ for each $d \geq 5$ such that $\mathcal{A}^{d} \subset \mathbb{N}^{d}$ is $\Delta$-normal, cone $\left(\mathcal{A}^{d}\right)$ is not simplicial and $\mathcal{A}^{d}$ has no unimodular triangulations. For each $d \geq 5$ let $\mathbf{p}_{d}=\mathbf{e}_{1}+\cdots+\mathbf{e}_{4} \in \mathbb{Z}^{d}, \mathbf{p}_{d}^{+}=\mathbf{p}_{d}+\mathbf{e}_{d} \in \mathbb{Z}^{d}$ 
and $\mathbf{p}_{d}^{-}=\mathbf{p}_{d}-\mathbf{e}_{d} \in \mathbb{Z}^{d}$. Here $\mathbf{e}_{d}$ is the $d$-th unit vector in $\mathbb{R}^{d}$. Letting $\mathcal{A}^{4}:=\mathcal{A}$ (a Firla-Ziegler configuration in $\left.\mathbb{N}^{4}\right)$, recursively define $\mathcal{A}^{d-1^{\prime}}:=\left\{(\mathbf{a}, 0): \mathbf{a} \in \mathcal{A}^{d-1}\right\}$ and $\mathcal{A}^{d}:=\mathcal{A}^{d-1^{\prime}} \cup\left\{\mathbf{p}_{d}^{+}, \mathbf{p}_{d}^{-}\right\}$. We assume that $\mathbf{p}_{d}^{+}$and $\mathbf{p}_{d}^{-}$are always the second last and last elements of $\mathcal{A}^{d}$. Let $\mathcal{A}^{d}$ have $n_{d}$ elements. We now construct a regular triangulation $\Delta^{d}$ of $\mathcal{A}^{d}$ as follows:

Suppose the first four columns of $\mathcal{A}^{4}$ are $\mathbf{e}_{1}, \mathbf{e}_{2}, \mathbf{e}_{3}, \mathbf{v}$. Let $\Delta^{4}$ be the coarse regular triangulation of $\mathcal{A}^{4}$ (and $\mathcal{A}^{4}$ ) consisting of the unique simplex $\sigma=\{1,2,3,4\}$. It can be induced with a weight vector $\mathbf{w}^{4}=(0,0,0,0, M, M, \ldots, M, M) \in \mathbb{Z}^{n_{4}}$ where $M$ is a large positive integer. We want $\Delta^{5}$ to consist of the simplices $\sigma_{1}:=\sigma \cup\left\{n_{5}-1\right\}$ and $\sigma_{2}:=\sigma \cup\left\{n_{5}\right\}$. This triangulation can be induced by the weight vector $\mathbf{w}^{5}=\left(\mathbf{w}^{4}, 1,1\right) \in \mathbb{Z}^{n_{5}}$. We repeat this construction for $d=6$ to get the regular triangulation $\Delta^{6}$ of $\mathcal{A}^{6}$ consisting of the simplices

$$
\sigma_{1} \cup\left\{n_{6}-1\right\}, \sigma_{2} \cup\left\{n_{6}-1\right\}, \sigma_{1} \cup\left\{n_{6}\right\} \text { and } \sigma_{2} \cup\left\{n_{6}\right\} .
$$

This can again be achieved by the weight vector $\mathbf{w}^{6}=\left(\mathbf{w}^{5}, 2,2\right) \in \mathbb{Z}^{n_{6}}$ provided $M$ is still big enough. In general we get the regular triangulation $\Delta^{d}$ of $\mathcal{A}^{d}$ consisting of the simplices:

$$
\left\{\sigma_{1}=\sigma \cup\left\{n_{d}-1\right\}, \forall \sigma \in \max \Delta^{d-1}\right\} \cup\left\{\sigma_{2}=\sigma \cup\left\{n_{d}\right\}, \forall \sigma \in \max \Delta^{d-1}\right\} .
$$

Let $K_{1}$ be a maximal subcone of $\operatorname{cone}\left(\mathcal{A}^{d}\right)$ of the form $K_{1}=\operatorname{cone}\left(\mathcal{A}_{\sigma_{1}}^{d}\right)$ and similarly let $K_{2}$ be a maximal cone of the form $K_{2}=\operatorname{cone}\left(\mathcal{A}_{\sigma_{2}}^{d}\right)$ induced by the regular triangulation $\Delta^{d}$. For $d=5$ we have precisely two cones, one of the form $K_{1}$, the other $K_{2}$ but for $d \geq 6$ there are several cones of types $K_{1}$ and $K_{2}$.

Lemma 5.3 The configuration $\mathcal{A}^{5}$ has the following properties:

(1) $\mathbb{Z}\left(\mathcal{A}^{5} \cap K_{1}\right)=\mathbb{Z}\left(\mathcal{A}^{5} \cap K_{2}\right)=\mathbb{Z}^{5}$,

(2) $\mathcal{A}^{5}$ is non-simplicial,

(3) $\mathcal{A}^{5}$ is $\Delta^{5}$-normal, and

(4) $\mathcal{A}^{5}$ admits no unimodular triangulations.

\section{Proof:}

(1) Since $\mathbf{p}_{5}=(1,1,1,1,0), \mathbf{p}_{5}^{+}=(1,1,1,1,1)$ and the first three unit vectors of $\mathbb{R}^{5}$ belong to $\mathcal{A}^{5} \cap K_{1}$, it follows that all unit vectors of $\mathbb{R}^{5}$ lie in $\mathbb{Z}\left(\mathcal{A}^{5} \cap K_{1}\right)$ which gives the result. Similarly, $\mathbb{Z}\left(\mathcal{A}^{5} \cap K_{2}\right)=\mathbb{Z}^{5}$.

(2) Since $\mathbf{p}_{5}$ lies in the interior of cone $\left(\mathcal{A}^{5}\right)$, the vectors $\mathbf{p}_{5}^{+}$and $\mathbf{p}_{5}^{-}$do not lie on a common facet of the cone. Hence cone $\left(\mathcal{A}^{5}\right)$ is a bipyramidal cone over cone $\left(\mathcal{A}^{4^{\prime}}\right)$ with six extreme rays and is hence non-simplicial.

(3) The triangulation $\Delta^{5}$ is a regular triangulation of $\mathcal{A}^{5}$. We first argue that $\mathcal{A}^{5} \cap K_{1}$ is a minimal generating set of the semigroup $K_{1} \cap \mathbb{Z}\left(\mathcal{A}^{5} \cap K_{1}\right) \stackrel{(1)}{=} K_{1} \cap \mathbb{Z}^{5}$. Suppose $\mathbf{q}=\left(q_{1}, \ldots, q_{5}\right) \in K_{1} \cap \mathbb{Z}^{5}$. Since $\mathbf{p}_{5}^{+}$is the unique generator of $K_{1}$ with a positive fifth co-ordinate, $\mathbf{q}=q_{5} \mathbf{p}_{5}^{+}+\mathbf{q}^{\prime}$ where $\mathbf{q}=\left(q_{1}-q_{5}, q_{2}-q_{5}, q_{3}-q_{5}, q_{4}-q_{5}, 0\right)^{t}$ is the unique expression of $\mathbf{q}$ as an $\mathbb{R}_{\geq 0}$-combination of $\mathbf{p}_{5}^{+}$and the other extreme rays of $K_{1}$. 
Since $q_{5}^{\prime}=0$, in fact, $\mathbf{q}^{\prime} \in \operatorname{cone}\left(\mathcal{A}^{4^{\prime}}\right) \cap \mathbb{Z}^{5} \stackrel{*}{=} \mathbb{N} \mathcal{A}^{4^{\prime}} \subset \mathbb{N}\left(K_{1} \cap \mathcal{A}^{5}\right)$ where the equality (*) follows from the normality of $\mathcal{A}^{4}$. This in turn implies that $\mathbf{q}=q_{5} \mathbf{p}_{5}^{+}+\mathbf{q}^{\prime} \in \mathbb{N}\left(K_{1} \cap \mathcal{A}^{5}\right)$. (Note that $q_{5} \in \mathbb{N}$.) Similarly, $\mathcal{A}^{5} \cap K_{2}$ is its own Hilbert basis. Thus, $\mathcal{A}^{5}$ is $\Delta^{5}$-normal.

(4) Suppose $T$ is a unimodular triangulation of $\mathcal{A}^{5}$ and $\tau$ is a facet of $T$. Then by (1), $\left|\operatorname{det}\left(\mathbf{A}_{\tau}^{5}\right)\right|=1$ and $\left\{n_{5}-1, n_{5}\right\} \cap \tau \neq \emptyset$. If $\left\{n_{5}-1, n_{5}\right\} \subset \tau$, then

$$
\mathbf{A}_{\tau}^{5}=\left(\begin{array}{ccccc}
* & * & * & 1 & 1 \\
* & * & * & 1 & 1 \\
* & * & * & 1 & 1 \\
* & * & * & 1 & 1 \\
0 & 0 & 0 & 1 & -1
\end{array}\right)
$$

which shows that $\left|\operatorname{det}\left(\mathbf{A}_{\tau}^{5}\right)\right| \in 2 \mathbb{Z}$, a contradiction. Hence each maximal simplex in $T$ contains exactly one of $\mathbf{p}_{5}^{+}$or $\mathbf{p}_{5}^{-}$and $T$ induces a triangulation $T^{\prime}$ of $\mathcal{A}^{4^{\prime}}$. Since $T$ is unimodular, $T^{\prime}$ gives a unimodular triangulation of $\mathcal{A}^{4}$ which is a contradiction as $\mathcal{A}^{4}$ has no unimodular triangulations. Therefore, we conclude that $\mathcal{A}^{5}$ has no unimodular triangulations.

Theorem 5.4 For each $d \geq 5$, the configuration $\mathcal{A}^{d}$ has the following properties:

(1) $\mathbb{Z}\left(\mathcal{A}^{d} \cap K_{1}\right)=\mathbb{Z}\left(\mathcal{A}^{d} \cap K_{2}\right)=\mathbb{Z}^{d}$,

(2) $\mathcal{A}^{d}$ is non-simplicial,

(3) $\mathcal{A}^{d}$ is $\Delta^{d}$-normal, and

(4) $\mathcal{A}^{d}$ admits no unimodular triangulations.

Proof: This theorem is proved by induction using Lemma 5.3 as the base step.

(1) Suppose the result is true for $k=d-1$. Then it follows that $\mathbb{Z} \mathcal{A}^{d-1^{\prime}}$ contains the first $d-1$ unit vectors of $\mathbb{Z}^{d}$ which are therefore also in $\mathbb{Z}\left(\mathcal{A}^{d} \cap K_{1}\right)$ and $\mathbb{Z}\left(\mathcal{A}^{d} \cap K_{2}\right)$. Since $\mathbf{p}_{d}^{+} \in K_{1} \cap \mathcal{A}^{d}$ (and $\left.\mathbf{p}_{d}^{-} \in K_{2} \cap \mathcal{A}^{d}\right)$, we also get that $\mathbf{e}_{d} \in \mathbb{Z}\left(\mathcal{A}^{d} \cap K_{1}\right)$ (and $\left.\mathbf{e}_{d} \in \mathbb{Z}\left(\mathcal{A}^{d} \cap K_{2}\right)\right)$. Hence $\mathbb{Z}\left(\mathcal{A}^{d} \cap K_{1}\right)=\mathbb{Z}\left(\mathcal{A}^{d} \cap K_{2}\right)=\mathbb{Z}^{d}$.

(2) Assume by induction that $\mathcal{A}^{d-1}$ is non-simplicial and that $\mathbf{p}_{d-1}$ lies in the interior of cone $\left(\mathcal{A}^{d-1}\right)$. Then, $\mathbf{p}_{d}$ lies in the interior of cone $\left(\mathcal{A}^{d}\right)$ and hence $\mathbf{p}_{d}^{+}$and $\mathbf{p}_{d}^{-}$do not lie on a common facet of cone $\left(\mathcal{A}^{d}\right)$. This implies that cone $\left(\mathcal{A}^{d}\right) \subset \mathbb{R}^{d}$ has exactly two more extreme rays than cone $\left(\mathcal{A}^{d-1}\right) \subset \mathbb{R}^{d-1}$ and hence is non-simplicial.

(3) As in Lemma 5.3, $\Delta^{d}$ is a regular triangulation of $\mathcal{A}^{d}$ for each $d \geq 5$. We assume by induction that $\mathcal{A}^{d-1}$ is $\Delta^{d-1}$-normal and hence normal. The arguments that $\mathcal{A}^{d} \cap K_{1}$ and $\mathcal{A}^{d} \cap K_{2}$ are minimal generating sets for $K_{1} \cap \mathbb{Z}^{d}$ and $K_{2} \cap \mathbb{Z}^{d}$ respectively follow from a straight generalization of the arguments in Lemma 5.3.

(4) Again we assume by induction that $\mathcal{A}^{d-1}$ admits no unimodular triangulations. The rest of the argument is also a straight generalization of the arguments in Lemma 5.3 (4). 
We have thus produced non-simplicial $\Delta$-normal configurations without unimodular triangulations in every dimension beyond four, starting with a Firla-Ziegler $\mathcal{A}$ in $\mathbb{N}^{4}$. The construction applies to all such Firla-Ziegler configurations.

Every normal configuration $\mathcal{A}$ in dimension $d \leq 3$ admits a unimodular triangulation and thus the only remaining dimension in which there might be non-simplicial $\Delta$-normal configurations without unimodular triangulations is dimension four. Here is an example.

Example 5.2 (continued) Consider the matrix $\mathbf{B}$ below obtained by appending the column $(1,1,1,2)$ to the matrix $\mathbf{A}$ from Example 5.2.

$$
\mathbf{B}=\left(\begin{array}{lllllllll}
1 & 0 & 0 & 1 & 1 & 1 & 1 & 1 & 1 \\
0 & 1 & 0 & 2 & 1 & 1 & 2 & 2 & 1 \\
0 & 0 & 1 & 3 & 1 & 2 & 2 & 3 & 1 \\
0 & 0 & 0 & 5 & 1 & 2 & 3 & 4 & 2
\end{array}\right) .
$$

The configuration $\mathcal{B}$ given by the columns of $\mathbf{B}$ generate a four dimensional, non-simplicial cone with extreme rays generated by columns $1,2,3,4$, and 9 . Columns 3 and 9 lie on opposite sides of the linear span of columns 1, 2 and 4 , and the determinant of the submatrix of $\mathbf{B}$ consisting of columns $1,2,4$, and 9 is one. The weight vector $(0,0,0,0,10,10,10,10,1)$ induces the regular triangulation $\Delta$ of $\mathcal{B}$ with maximal simplices $\{1,2,3,4\}$ and $\{1,2,4,9\}$. The configuration $\mathcal{B}$ is $\Delta$-normal by construction. Further, this configuration has no regular unimodular triangulations (confirmed using CaTS [6]).

\subsection{Non $\Delta$-normal configurations from an example of Hibi and Ohsugi}

Consider the graph $G_{\mathrm{HO}}$ shown in figure 2. In [8] Hibi and Ohsugi showed that the graded normal configuration

$$
\mathcal{A}_{\mathrm{HO}}=\left\{\mathbf{e}_{1}+\mathbf{e}_{i}+\mathbf{e}_{j}:\{i, j\} \in E\left(G_{\mathrm{HO}}\right), 1 \notin\{i, j\}\right\} \cup\left\{\mathbf{e}_{1}+\mathbf{e}_{i}:\{1, i\} \in E\left(G_{\mathrm{HO}}\right)\right\}
$$

admits no regular unimodular triangulations, although it does have non-regular unimodular triangulations. Further, the 15 points in $\mathcal{A}_{\mathrm{HO}}$ are all extreme points of the convex hull of

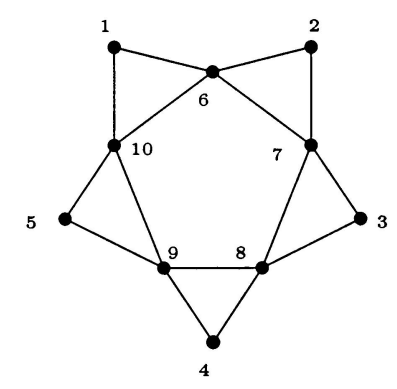

Figure 2. The graph $G_{H O}$ giving the Hibi-Ohsugi configuration. 
$\mathcal{A}_{\mathrm{HO}}$, denoted as $\operatorname{conv}\left(\mathcal{A}_{\mathrm{HO}}\right)$. Similar constructions of normal configurations arising from graphs that have no regular unimodular triangulations are given in [14]. The $(0,1)$-polytope $\operatorname{conv}\left(\mathcal{A}_{\mathrm{HO}}\right) \subset\left\{\mathbf{x} \in \mathbb{R}^{10}: x_{1}=1\right\}$ is empty which means that it has no lattice points other than its vertices. Thus cone $\left(\mathcal{A}_{\mathrm{HO}}\right)$ which is the cone over $\operatorname{conv}\left(\mathcal{A}_{\mathrm{HO}}\right)$ has 15 extreme rays and is therefore non-simplicial.

Lemma 5.5 Let $\mathcal{A} \subset \mathbb{Z}^{d}$ be a normal graded non-simplicial configuration in $\left\{\mathbf{x} \in \mathbb{R}^{d}\right.$ : $\left.x_{1}=1\right\}$ such that $\operatorname{conv}(\mathcal{A})$ is empty. If $\mathcal{A}$ does not have a regular unimodular triangulation then $\mathcal{A}$ is not $\Delta$-normal for any regular triangulation $\Delta$.

Proof: Without loss of generality, we can assume that $\mathbb{Z} \mathcal{A}=\mathbb{Z}^{d}$. By the hypothesis, every regular triangulation $\Delta$ of $\mathcal{A}$ has a maximal face $\sigma$ such that $\left|\operatorname{det}\left(\mathbf{A}_{\sigma}\right)\right| \geq 2$. Thus the Hilbert basis of cone $\left(\mathcal{A}_{\sigma}\right)$ contains at least one vector $\mathbf{q} \in \mathbb{Z}^{d}$ not in $\mathcal{A}_{\sigma}$. Since all vectors in $\mathcal{A}$ are extreme rays of cone $(\mathcal{A})$, none of them lie in cone $\left(\mathcal{A}_{\sigma}\right)$ unless they are in $\mathcal{A}_{\sigma}$. This implies that $\mathcal{A}_{\sigma}=\operatorname{cone}\left(\mathcal{A}_{\sigma}\right) \cap \mathcal{A}$ is not normal and hence $\mathcal{A}$ is not $\Delta$-normal.

Corollary 5.6 The Hibi-Ohsugi configuration $\mathcal{A}_{\mathrm{HO}}$ is not $\Delta$-normal for any regular triangulation $\Delta$.

From $\mathcal{A}_{\mathrm{HO}}$ we now recursively construct configurations $\mathcal{A}^{d}$ for each $d \geq 11$ such that $\mathcal{A}^{d}$ is normal and graded but not $\Delta$-normal for any regular triangulation $\Delta$. For each $d \geq 11$ let $\mathbf{p}_{d}=\mathbf{e}_{1}+\mathbf{e}_{d} \in \mathbb{Z}^{d}$. Letting $\mathcal{A}^{10}:=\mathcal{A}$, recursively define

$$
\mathcal{A}^{d-1^{\prime}}:=\left\{\left(\begin{array}{l}
\mathbf{a} \\
0
\end{array}\right): \mathbf{a} \in \mathcal{A}^{d-1}\right\} \quad \text { and } \quad \mathcal{A}^{d}:=\left\{\mathbf{p}_{d}\right\} \cup \mathcal{A}^{d-1^{\prime}} .
$$

Theorem 5.7 For each $d \geq 10$, the configuration $\mathcal{A}^{d}$ is normal and graded but not $\Delta$-normal for any regular triangulation $\Delta$.

Proof: It suffices to show that $\mathcal{A}^{d}$ satisfies the conditions of Lemma 5.5 for each $d$. For a given $d, \mathcal{A}^{d}$ is graded since it lies in $\left\{\mathbf{x} \in \mathbb{R}^{d}: x_{1}=1\right\}$ and $\operatorname{conv}\left(\mathcal{A}^{d}\right)$ is a $(0,1)$-polytope and hence empty. Further, cone $\left(\mathcal{A}^{d}\right)$ is non-simplicial as cone $\left(\mathcal{A}^{d-1}\right)$ is non-simplicial for all $d \geq 11$.

The configuration $\mathcal{A}^{11}$ is normal. To see this let $\mathbf{q}:=\left(q_{1}, \cdots, q_{11}\right)^{t} \in \operatorname{cone}\left(\mathcal{A}^{11}\right) \cap \mathbb{Z} \mathcal{A}^{11}$. Since the only extreme ray of cone $\left(\mathcal{A}^{11}\right)$ with non-zero eleventh co-ordinate is $\mathbf{p}_{11}, q_{11} \leq q_{1}$. Further, $\mathbf{q}=q_{11} \mathbf{p}_{11}+\mathbf{q}^{\prime}$, where $\mathbf{q}^{\prime}=\left(q_{1}-q_{11}, q_{2}, \cdots, q_{10}, 0\right)^{t}$, is the unique expression of $\mathbf{q}$ as an $\mathbb{R}_{\geq 0}$-combination of the extreme rays of cone $\left(\mathcal{A}^{11}\right)$. The integral vector $\mathbf{q}^{\prime}$ lies in $\mathbb{N} \mathcal{A}^{10^{\prime}}$ since $\mathcal{A}^{10}$ is normal and hence it lies in $\mathbb{N} \mathcal{A}^{11}$. Thus $\mathbf{q} \in \mathbb{N} \mathcal{A}^{11}$. By induction, it follows that $\mathcal{A}^{d}$ is normal for all $d \geq 11$.

Suppose $\mathcal{A}^{11}$ had a regular unimodular triangulation. Then $\mathbf{p}_{11}$ would be a vertex in every maximal face of this regular unimodular triangulation of $\operatorname{conv}(\mathcal{A})$. This in turn induces a regular unimodular triangulation in $\mathcal{A}^{10^{\prime}}$ and hence in $\mathcal{A}^{10}$, a contradiction. Again, a straightforward inductive argument shows that $\mathcal{A}^{d}$ has no regular unimodular triangulation for all $d \geq 11$. 
We have thus produced graded normal configurations that are not $\Delta$-normal for any regular triangulation $\Delta$ in every dimension $d \geq 10$.

Remark 5.8 A second infinite family of normal, graded, non-simplicial configurations without regular unimodular triangulations, based on $G_{\mathrm{HO}}$, can be found in [14]. The convex hulls of these configurations are all empty since they are all 0,1 -configurations. Thus by Lemma 5.5, they provide another infinite family of non- $\Delta$-normal configurations.

Example 5.2 (continued) Non- $\Delta$-normal configurations also exist in lower dimensions. The columns of each of the following matrices are two such examples (confirmed using CaTS [6]).

$$
\left(\begin{array}{ccccccccc}
1 & 0 & 0 & 1 & 1 & 1 & 1 & 1 & 0 \\
0 & 1 & 0 & 2 & 1 & 2 & 2 & 1 & 0 \\
0 & 0 & 1 & 3 & 2 & 2 & 3 & 1 & 0 \\
0 & 0 & 0 & 5 & 2 & 3 & 4 & 1 & 0 \\
0 & 0 & 0 & 0 & 0 & 0 & 0 & 1 & -1
\end{array}\right), \quad\left(\begin{array}{ccccccccc}
1 & 1 & 1 & 1 & 1 & 1 & 1 & 1 & 1 \\
0 & 1 & 0 & 0 & 1 & 1 & 1 & 1 & 1 \\
0 & 0 & 1 & 0 & 2 & 1 & 1 & 2 & 2 \\
0 & 0 & 0 & 1 & 3 & 1 & 2 & 2 & 3 \\
0 & 0 & 0 & 0 & 5 & 1 & 2 & 3 & 4
\end{array}\right)
$$

\section{Acknowledgments}

We thank Diane Maclagan for alerting us to the interpretation of Simon's condition for a monomial ideal to be Cohen-Macaulay in her paper [12] with Greg Smith. We also thank Winfried Bruns, Joseph Gubeladze, Serkan Hoşten, Francisco Santos and Günter Ziegler for discussions on constructing $\Delta$-normal configurations. Finally, we thank the referee for his/her thoughtful and constructive suggestions. Research partially supported by NSF grant DMS 0100141. The first author is partially supported by a Fulbright grant.

\section{References}

1. W. Bruns, J. Gubeladze, and N.V. Trung, "Normal polytopes, triangulations, and Koszul algebras," J. Reine Angew. Math. 485 (1997), 123-160.

2. W. Bruns, J. Gubeladze, and N.V. Trung, "Problems and algorithms for affine semigroups," Semigroup Forum 64(2) (2002), 180-212.

3. W. Bruns and R. Koch, "Computing the integral closure of an affine semigroup, Effective methods in algebraic and analytic geometry, 2000 (Krakow)," Univ. Iagel. Acta Math. 39 (2001), 59-70. Software: Normaliz, ftp.mathematik.Uni-Osnabrueck.DE/pub/osm/kommalg/software.

4. R.T. Firla, private correspondence.

5. R.T. Firla and G.M. Ziegler, "Hilbert bases, unimodular triangulations, and binary covers of rational polyhedral cones," Discrete Comp. Geom. 21 (1999), 205-216.

6. A. Jensen, "CaTS, a software package for computing state polytopes of toric ideals," available from http://www.soopadoopa.dk/anders/cats/cats.html.

7. D. Grayson and M. Stillman, "Macaulay 2, a software system for research in algebraic geometry," available from http://www.math.uiuc.edu/Macaulay2/.

8. T. Hibi and H. Ohsugi, "A normal $(0,1)$-polytope none of whose regular triangulations are unimodular," Discrete Comp. Geom. 21 (1999), 201-204. 
9. M. Hochster, "Rings of invariants of tori, Cohen-Macaulay rings generated by monomials, and polytopes," Ann. Math. 96 (1972), 318-337.

10. S. Hoşten, D. Maclagan, and B. Sturmfels, "Supernormal vector configurations," to appear in J. Algebraic Combin., math.CO/0105036.

11. S. Hoşten and R.R.Thomas, "Gomory integer programs," Math. Programming Series B 96 (2003), 271-292.

12. D. Maclagan and G.G. Smith, "Uniform bounds on multigraded regularity," J. Algebraic Geom. (to appear).

13. L. Matusevich, private correspondence.

14. H. Ohsugi, "Toric ideals and an infinite family of normal $(0,1)$-polytopes without unimodular regular triangulations," Discrete Comp. Geom. 27 (2002), 551-565.

15. R.S. Simon, "Combinatorial Properties of 'cleanness'," J. Algebra 167 (1994), 361-368.

16. R. Stanley, Combinatorics and Commutative Algebra, 2nd edition, Progress in Mathematics, vol. 41, Birkhäuser Boston, Inc., Boston, MA, 1996.

17. B. Sturmfels, Gröbner Bases and Convex Polytopes, University Lecture Series, vol. 8, American Mathematical Society, Providence, RI, 1996.

18. B. Sturmfels, "Equations defining toric varieties, Algebraic geometry-Santa Cruz," Proc. Sympos. Pure Math. 62(2) (1995), 437-449. Amer. Math. Soc., Providence, RI, 1997.

19. B. Sturmfels, N.V. Trung, and W. Vogel,"Bounds on projective schemes," Math. Ann. 302 (1995), 417-432.

20. G.M. Ziegler, Lectures on Polytopes, Graduate Texts in Mathematics, vol. 152, Springer-Verlag, New York, 1995. 